\title{
A convenient procedure for the catalytic asymmetric 1,3-dipolar cycloaddition of azomethine ylides and alkenes
}

Carlos Alemparte, ${ }^{\mathrm{a}}$ Gonzalo Blay, ${ }^{\mathrm{a}, \mathrm{b}}$ Karl Anker Jørgensen ${ }^{\mathrm{a} *}$

${ }^{a}$ Danish National Research Foundation: Center for Catalysis, Department of Chemistry, Aarhus University, DK-8000 Aarhus C, Denmark and ${ }^{b}$ Departament de Química Orgànica, Facultat de Química, Universitat de València, Dr. Moliner, 50, 46100-Burjassot (València), Spain

General procedures. ${ }^{1} \mathrm{H}$ NMR and ${ }^{13} \mathrm{C}$ NMR spectra were recorded in $\mathrm{CDCl}_{3}$ at 400 $\mathrm{MHz}$ and $100 \mathrm{MHz}$, respectively. Chemical shifts are reported in ppm $(\delta)$ relative to residual protic solvent ( $\delta 7.26$ for ${ }^{1} \mathrm{H}$ NMR and $\delta 77.0$ for ${ }^{13} \mathrm{C}$ NMR, respectively). Column chromatography was carried out using Fluka silica gel 60 (0.04-0.063 mm particle size). Thin layer chromatography (TLC) was performed on Merck silica gel 60 $\mathrm{F}_{254}$ plates and visualized by UV-light $(254 \mathrm{~nm})$ or by an aqueous mixture of $\mathrm{KMnO}_{4}$, $\mathrm{K}_{2} \mathrm{CO}_{3}$ and $\mathrm{NaOH}$. Optical rotations were measured on a Perkin-Elmer 241 polarimeter in dichloromethane solutions. The enantiomeric excess of the products was determined by HPLC using Daicel Chiralpack AS columns with 2-propanol/hexane mixtures as eluent. HRMS were recorded on a Micromass LC-TOF instrument.

Reagents and materials. $\mathrm{CH}_{2} \mathrm{Cl}_{2} \mathrm{HPLC}$ grade (Chromasolv, Sigma-Aldrich) was used without further treatment. All reagents were used as purchased.

Representative procedure for the preparation of the imines. To a suspension of 5.78 g (46 mmol) glycine methyl ester hydrochloride in $\mathrm{CH}_{2} \mathrm{Cl}_{2}(70 \mathrm{mmol})$ was added $10 \mathrm{~g}$ anhydrous $\mathrm{MgSO}_{4}$ and $7 \mathrm{~mL}(50 \mathrm{mmol})$ of $\mathrm{Et}_{3} \mathrm{~N}$. The mixture was stirred for $1 \mathrm{~h}$ at room 
temperature. Then the aldehyde $(40 \mathrm{mmol})$ was added and the resulting mixture stirred overnight. The reaction mixture was filtered and the organic phase washed with water (2 x $50 \mathrm{~mL}$ ) and dried over anhydrous $\mathrm{MgSO}_{4}$, filtered and evaporated under reduced pressure. The imines were used without further purification.

General procedure for the silver fluoride-cinchonine catalyzed 1,3-dipolar cycloaddition. Silver fluoride $(2.5 \mathrm{mg}, 0.02 \mathrm{mmol})$, hydrocinchonine $(5.9 \mathrm{mg}, 0.02$ mmol) and the imine $1(0.40 \mathrm{mmol})$ were placed in a test tube. $\mathrm{CH}_{2} \mathrm{Cl}_{2}(3.2 \mathrm{~mL})$ was added followed by tert-butyl acrylate $(70 \mu \mathrm{L}, 0.48 \mathrm{mmol})$. The tube was stopped with a rubber septum and stirred in a freezer at $-25^{\circ} \mathrm{C}$ for 4 days. After this time, the reaction mixture was chromatographed on silica gel using hexane-EtOAc or dichloromethanediethyl ether mixtures containing 10 drops $\mathrm{Et}_{3} \mathrm{~N} / 100 \mathrm{~mL}$ solvent to afford pyrrolidine 3 .

tert-Butyl $\quad(2 S, \quad 4 S, \quad 5 R)$-2-methoxycarbonyl-5-(4-methylphenyl)pyrrolidine-4carboxylate (3c).

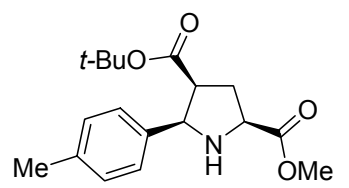

Obtained after purification by column chromatography using $\mathrm{CH}_{2} \mathrm{Cl}_{2}-\mathrm{Et}_{2} \mathrm{O}(95: 5)$ in $89 \%$ yield and $70 \%$ ee (Chiralpack AS, hexane/2-propanol 99:1, flow rate $1 \mathrm{~mL} / \mathrm{min}, \mathrm{t}_{\mathrm{R}(\text { major })}=12.9 \mathrm{~min}$, $\left.\mathrm{t}_{\mathrm{R}(\text { minor })}=26.3 \mathrm{~min}\right) . \quad[\alpha]_{\mathrm{D}}^{25}+23 \quad\left(\mathrm{c} \quad 0.53, \mathrm{CH}_{2} \mathrm{Cl}_{2}\right) ;$ HRMS $\mathrm{C}_{18} \mathrm{H}_{25} \mathrm{NO}_{4}[\mathrm{M}+\mathrm{Na}]^{+}$calculated 342.1676, found 342.1674; ${ }^{1} \mathrm{H}$ NMR (400 MHz, $\left.\mathrm{CDCl}_{3}\right) \delta 7.21(2 \mathrm{H}, \mathrm{d}, J=8.0 \mathrm{~Hz}), 7.09(2 \mathrm{H}, \mathrm{d}, J=8.0 \mathrm{~Hz}), 4.42(1 \mathrm{H}, \mathrm{d}, J=7.6 \mathrm{~Hz})$, $3.91(1 \mathrm{H}, \mathrm{t}, J=8.4 \mathrm{~Hz}), 3.79(3 \mathrm{H}, \mathrm{s}), 3.21(1 \mathrm{H}, \mathrm{dd}, J=14.4,8.0 \mathrm{~Hz}), 2.81(1 \mathrm{H}, \mathrm{br} \mathrm{s})$, 2.45-2.39 (1H, m), $2.30(3 \mathrm{H}, \mathrm{s}), 2.31-2.23(1 \mathrm{H}, \mathrm{m}), 1.02(9 \mathrm{H}, \mathrm{s}) ;{ }^{13} \mathrm{C} \mathrm{NMR}(100 \mathrm{MHz}$, $\left.\mathrm{CDCl}_{3}\right) \delta 173.7,171.9,136.8,136.1,128.6(2 \mathrm{C}), 127.0(2 \mathrm{C}), 80.4,65.3,59.8,52.2$, $50.2,34.1,27.4(3 \mathrm{C}), 21.0$.

tert-Butyl $\quad(2 S, \quad 4 S, \quad 5 R)$-2-methoxycarbonyl-5-(2-methylphenyl)pyrrolidine-4carboxylate (3d).

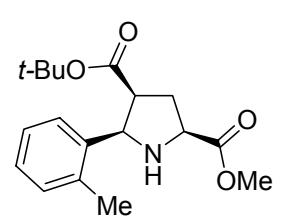

Obtained after purification by column chromatography using $\mathrm{CH}_{2} \mathrm{Cl}_{2}-\mathrm{Et}_{2} \mathrm{O}$ (95:5) in $97 \%$ yield and $64 \%$ ee (Chiralpack $\mathrm{AS}$, hexane/2-propanol 95:5, flow rate $1 \mathrm{~mL} / \mathrm{min}, \mathrm{t}_{\mathrm{R}(\text { major) }}=5.6 \mathrm{~min}$, $\left.\mathrm{t}_{\mathrm{R}(\text { minor })}=21.2 \mathrm{~min}\right) .[\alpha]^{25}+48\left(\right.$ c $\left.2.4, \mathrm{CH}_{2} \mathrm{Cl}_{2}\right) ;{ }^{1} \mathrm{H}$ NMR and ${ }^{13} \mathrm{C}$

NMR were consistent with previously reported values for its enantiomer. ${ }^{1}$ 
tert-Butyl (2S, $4 S, 5 R)$-2-methoxycarbonyl-5-phenylpyrrolidine-4-carboxylate (3e).

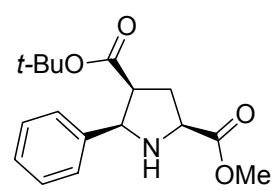

Obtained after purification by column chromatography using hexane-EtOAc $(75: 25)$ in $88 \%$ yield and $64 \%$ ee (Chiralpack AS, hexane/2-propanol 95:5, flow rate $1.0 \mathrm{~mL} / \mathrm{min}, \mathrm{t}_{\mathrm{R}(\text { major })}=7.9 \mathrm{~min}$, $\left.\mathrm{t}_{\mathrm{R}(\text { minor })}=12.5 \mathrm{~min}\right) .[\alpha]^{25}{ }_{\mathrm{D}}+24\left(c 0.86, \mathrm{CH}_{2} \mathrm{Cl}_{2}\right) ;{ }^{1} \mathrm{H} \mathrm{NMR}$ and ${ }^{13} \mathrm{C}$ NMR were consistent with previously reported values for its enantiomer. ${ }^{1}$

tert-Butyl $\quad(2 S, 4 S, 5 R)$-2-methoxycarbonyl-5-(2-methoxylphenyl)pyrrolidine-4carboxylate (3f).

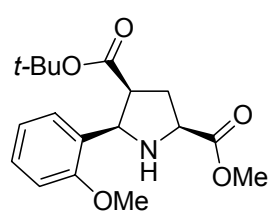

Obtained after purification by column chromatography using hexaneEtOAc (65:35) in 63\% yield and 56\% ee (Chiralpack AS, hexane/2propanol 99:1, flow rate $1 \mathrm{~mL} / \mathrm{min}, \mathrm{t}_{\mathrm{R}(\text { major })}=14.5 \mathrm{~min}, \mathrm{t}_{\mathrm{R}(\operatorname{minor})}=28.5$ $\min$ ). $[\alpha]_{\mathrm{D}}^{25}+67$ (c $0.41, \mathrm{CH}_{2} \mathrm{Cl}_{2}$ ); HRMS $\mathrm{C}_{18} \mathrm{H}_{25} \mathrm{NO}_{5}[\mathrm{M}+\mathrm{Na}]^{+}$ calculated 358.1625, found 358.1623; ${ }^{1} \mathrm{H} \mathrm{NMR}\left(400 \mathrm{MHz}, \mathrm{CDCl}_{3}\right) \delta 7.33(1 \mathrm{H}, \mathrm{d}, J=$ $8.0 \mathrm{~Hz}), 7.24, \mathrm{t}, J=8.0 \mathrm{~Hz}), 6.93(1 \mathrm{H}, \mathrm{t}, J=8.0 \mathrm{~Hz}), 6.84(1 \mathrm{H}, \mathrm{d}, J=8.0 \mathrm{~Hz}), 4.51(1 \mathrm{H}$, $\mathrm{d}, J=8.4 \mathrm{~Hz}), 3.90(1 \mathrm{H}, \mathrm{t}, J=8.4 \mathrm{~Hz}), 3.85(3 \mathrm{H}, \mathrm{s}), 3.81(3 \mathrm{H}, \mathrm{s}), 3.43(1 \mathrm{H}, \mathrm{td}, J=8.4$, $4.0 \mathrm{~Hz}), 2.91\left(1 \mathrm{H}\right.$, br s), 2.29-2.23 (1H, m), $0.98(9 \mathrm{H}, \mathrm{s}) ;{ }^{13} \mathrm{C} \mathrm{NMR}\left(100 \mathrm{MHz}, \mathrm{CDCl}_{3}\right) \delta$ $173.5,172.8,156.8,128.2$, 126.6, 126.4, 120.2, 109.7, 79.9, 61.6, 59.6, 55.1, 52.1, 47.8, $34.5,27.4(3 \mathrm{C})$.

tert-Butyl $\quad(2 S, 4 S, \quad 5 R)$-2-methoxycarbonyl-5-(3-methoxylphenyl)pyrrolidine-4carboxylate (3g).

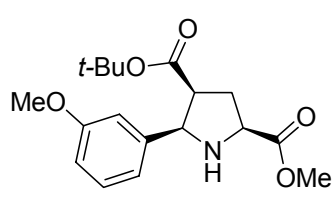

Obtained after purification by column chromatography using hexane-EtOAc (65:35) in 93\% yield and 70\% ee $(2 \times$ Chiralpack

AS, hexane/2-propanol 99:1, flow rate $1 \mathrm{~mL} / \mathrm{min}, \mathrm{t}_{\mathrm{R} \text { (major) }}=53.6$ $\left.\min , \mathrm{t}_{\mathrm{R}(\operatorname{minor})}=65.9 \mathrm{~min}\right) \cdot[\alpha]_{\mathrm{D}}^{25}+21$ (c 2.4, $\mathrm{CH}_{2} \mathrm{Cl}_{2}$ ); HRMS $\mathrm{C}_{18} \mathrm{H}_{25} \mathrm{NO}_{5}[\mathrm{M}+\mathrm{Na}]^{+}$calculated 358.1625, found 358.1630; ${ }^{1} \mathrm{H}$ NMR (400 MHz, $\left.\mathrm{CDCl}_{3}\right) \delta 7.20(1 \mathrm{H}, \mathrm{t}, J=8.0 \mathrm{~Hz}), 6.92(1 \mathrm{H}, \mathrm{d}, J=8.0 \mathrm{~Hz}), 6.91(1 \mathrm{H}, \mathrm{d}, J=2.8 \mathrm{~Hz})$, $6.77(1 \mathrm{H}, \mathrm{dd}, J=2.8,8.0 \mathrm{~Hz}), 4.43(1 \mathrm{H}, \mathrm{d}, J=8.0 \mathrm{~Hz}), 3.92(1 \mathrm{H}, \mathrm{t}, \mathrm{J}=8.4 \mathrm{~Hz}), 3.79$ $(3 \mathrm{H}, \mathrm{s}), 3.78(3 \mathrm{H}, \mathrm{s}), 3.23(1 \mathrm{H}, \mathrm{dd}, J=7.6,14.0 \mathrm{~Hz}), 2.80(1 \mathrm{H}, \mathrm{br} \mathrm{s}), 2.45-2.38(1 \mathrm{H}, \mathrm{m})$, 2.32-2.25 (1H, m), $1.04(9 \mathrm{H}, \mathrm{s}) ;{ }^{13} \mathrm{C} \mathrm{NMR}\left(100 \mathrm{MHz}, \mathrm{CDCl}_{3}\right) \delta 173.6,171.8,159.3$, $140.9,129.0,119.5,112.9,112.7,80.5,65.5,59.8,55.2,52.2,50.1,34.0,27.4(3 \mathrm{C})$. 
tert-Butyl $(2 S, 4 S, 5 R)$-2-methoxycarbonyl-5-(4-methoxylphenyl)pyrrolidine-4carboxylate (3h).

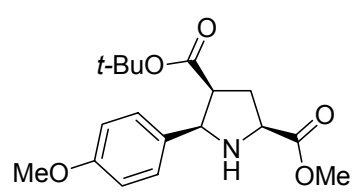

Obtained after purification by column chromatography using hexane-EtOAc $(65: 35)$ in $89 \%$ yield and $56 \%$ ee (Chiralpack $\mathrm{AS}$, hexane/2-propanol 95:5, flow rate $1 \mathrm{~mL} / \mathrm{min}, \mathrm{t}_{\mathrm{R}(\text { major })}=9.8$ $\left.\min , \mathrm{t}_{\mathrm{R}(\text { minor })}=18.5 \mathrm{~min}\right) .[\alpha]_{\mathrm{D}}^{25}+19\left(\right.$ c 2.1, $\left.\mathrm{CH}_{2} \mathrm{Cl}_{2}\right) ;{ }^{1} \mathrm{H} \mathrm{NMR}$ and ${ }^{13} \mathrm{C}$ NMR were consistent with previously reported values for its enantiomer. ${ }^{1}$

tert-Butyl $\quad(2 S, \quad 4 S, \quad 5 R)-2-m e t h o x y c a r b o n y l-5-(4-N, N$-dimethylaminophenyl)-4carboxylate (3i).

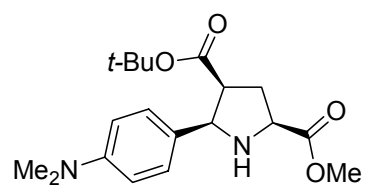

Obtained after purification by column chromatography using hexane-EtOAc $(65: 35)$ in $86 \%$ yield and $67 \%$ ee (Chiralpack $\mathrm{AS}$, hexane/2-propanol 99:1, flow rate $1 \mathrm{~mL} / \mathrm{min}, \mathrm{t}_{\mathrm{R}(\text { major })}=20.4$ $\left.\min , \mathrm{t}_{\mathrm{R}(\text { minor })}=36.9 \mathrm{~min}\right) .[\alpha]_{\mathrm{D}}^{25}+18\left(c \quad 0.53, \mathrm{CH}_{2} \mathrm{Cl}_{2}\right)$; HRMS $\mathrm{C}_{19} \mathrm{H}_{28} \mathrm{~N}_{2} \mathrm{O}_{4}[\mathrm{M}+\mathrm{Na}]^{+}$calculated 371.1941, found 371.1945; ${ }^{1} \mathrm{H}$ NMR (400 MHz, $\left.\mathrm{CDCl}_{3}\right) \delta 7.18(2 \mathrm{H}, \mathrm{d}, J=8.0 \mathrm{~Hz}), 6.67(2 \mathrm{H}, \mathrm{d}, J=8.0 \mathrm{~Hz}), 4.38(1 \mathrm{H}, \mathrm{d}, J=7.6 \mathrm{~Hz})$, $3.90(1 \mathrm{H}, \mathrm{t}, J=8.4 \mathrm{~Hz}), 3.79(3 \mathrm{H}, \mathrm{s}), 3.19(1 \mathrm{H}, \mathrm{dd}, J=7.6,14.0 \mathrm{~Hz}), 2.89(6 \mathrm{H}, \mathrm{s}), 2.60$ $\left(1 \mathrm{H}\right.$, br s), 2.42-2.35 $(1 \mathrm{H}, \mathrm{m}), 2.31-2.24(1 \mathrm{H}, \mathrm{m}), 1.05(9 \mathrm{H}, \mathrm{s}) ;{ }^{13} \mathrm{C}$ NMR $(100 \mathrm{MHz}$, $\left.\mathrm{CDCl}_{3}\right) \delta 173.7,171.9,150.0,127.8(2 \mathrm{C}), 127.3,112.4(2 \mathrm{C}), 80.3,65.1,59.8,52.1$, $50.4,40.7,34.1,27.5(3 \mathrm{C})$. Part of the product $(107 \mathrm{mg})$ was recrystallized from $5 \mathrm{~mL}$ of hexane and $1 \mathrm{~mL} \mathrm{CH}_{2} \mathrm{Cl}_{2}$ at $0{ }^{\circ} \mathrm{C}$. The mother liquors were concentrate to give $52 \mathrm{mg}$ ( $48 \%$ yield, $41 \%$ overall yield) of enantiomerically enriched product ( $92 \%$ ee).

tert-Butyl $\quad(2 S, \quad 4 S, \quad 5 R)$-2-methoxycarbonyl-5-(4-bromophenyl)pyrrolidine-4carboxylate $(3 \mathbf{j})$.

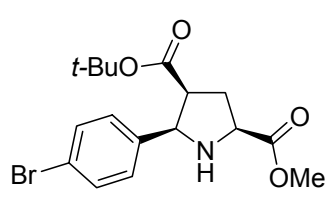

Obtained after purification by column chromatography using $\mathrm{CH}_{2} \mathrm{Cl}_{2}-\mathrm{Et}_{2} \mathrm{O}(80: 20)$ in $88 \%$ yield and $66 \%$ ee (Chiralpack AS, hexane/2-propanol 95:5, flow rate $1 \mathrm{~mL} / \mathrm{min}, \mathrm{t}_{\mathrm{R}(\text { major })}=7.8 \mathrm{~min}$, $\left.\mathrm{t}_{\mathrm{R}(\text { minor })}=16.2 \mathrm{~min}\right) .[\alpha]^{25}+18\left(c 0.76, \mathrm{CH}_{2} \mathrm{Cl}_{2}\right) ;{ }^{1} \mathrm{H} \mathrm{NMR}$ and ${ }^{13} \mathrm{C}$

NMR were consistent with previously reported values for its enantiomer. ${ }^{1}$ 
tert-Butyl $\quad(2 S, \quad 4 S, \quad 5 R)$-2-methoxycarbonyl-5-(4-cyanophenyl)pyrrolidine-4carboxylate (3k).

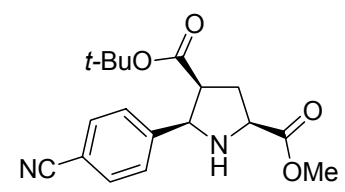

Obtained after purification by column chromatography using $\mathrm{CH}_{2} \mathrm{Cl}_{2}-\mathrm{Et}_{2} \mathrm{O}(80: 20)$ in $86 \%$ yield and $70 \%$ ee (Chiralpack AS, hexane/2-propanol 99:1, flow rate $1 \mathrm{~mL} / \mathrm{min}, \mathrm{t}_{\mathrm{R}(\text { major })}=25.8 \mathrm{~min}$, $\left.\mathrm{t}_{\mathrm{R}(\text { minor })}=42.9 \mathrm{~min}\right) .[\alpha]_{\mathrm{D}}^{25}+23\left(c 0.47, \mathrm{CH}_{2} \mathrm{Cl}_{2}\right) ;{ }^{1} \mathrm{H} \mathrm{NMR}$ and ${ }^{13} \mathrm{C}$ NMR were consistent with previously reported values for its enantiomer. ${ }^{1}$

tert-Butyl $\quad(2 S, \quad 4 S, \quad 5 R)-2$-methoxycarbonyl-5-(4-methoxycarbonylphenyl) pyrrolidine-4-carboxylate (3I).

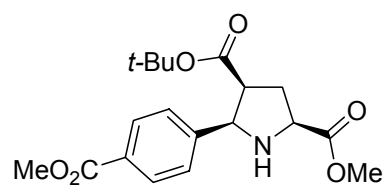

Obtained after purification by column chromatography using $\mathrm{CH}_{2} \mathrm{Cl}_{2}-\mathrm{Et}_{2} \mathrm{O}(80: 20)$ in $82 \%$ yield and $61 \%$ ee (Chiralpack AS, hexane/2-propanol 90:10, flow rate $1 \mathrm{~mL} / \mathrm{min}, \mathrm{t}_{\mathrm{R}(\text { major })}=9.0$ $\left.\min , \mathrm{t}_{\mathrm{R}(\text { minor })}=17.3 \mathrm{~min}\right) .[\alpha]_{\mathrm{D}}^{25}+20\left(\mathrm{c} 0.97, \mathrm{CH}_{2} \mathrm{Cl}_{2}\right)$; HRMS $\mathrm{C}_{19} \mathrm{H}_{25} \mathrm{NO}_{6}[\mathrm{M}+\mathrm{Na}]^{+}$calculated 386.1574, found 386.1579; ${ }^{1} \mathrm{H}$ NMR (400 MHz, $\left.\mathrm{CDCl}_{3}\right) \delta 7.99(2 \mathrm{H}, \mathrm{d}, J=8.0 \mathrm{~Hz}), 7.45(2 \mathrm{H}, \mathrm{d}, J=8.0 \mathrm{~Hz}), 4.50(1 \mathrm{H}, \mathrm{d}, J=8.0 \mathrm{~Hz})$, $3.96(1 \mathrm{H}, \mathrm{t}, J=8.0 \mathrm{~Hz}), 3.91(3 \mathrm{H}, \mathrm{s}), 3.81(3 \mathrm{H}, \mathrm{s}), 3.28(1 \mathrm{H}, \mathrm{dd}, J=7.0,14.0 \mathrm{~Hz}), 2.50-$ $2.42(1 \mathrm{H}, \mathrm{m}), 2.34-2.27(1 \mathrm{H}, \mathrm{m}), 1.02(9 \mathrm{H}, \mathrm{s}) ;{ }^{13} \mathrm{C} \mathrm{NMR}\left(100 \mathrm{MHz}, \mathrm{CDCl}_{3}\right) \delta 173.5$, 171.5, 166.8, 144.5, 129.4 (2C), 129.0, 127.2 (2C), 80.9, 65.2, 59.8, 52.3, 52.1, 49.9, $34.0,27.5$ (3C). Part of the obtained product $(113 \mathrm{mg})$ was recrystallized from $4 \mathrm{~mL}$ of hexane and $0.5 \mathrm{~mL} \mathrm{CH}_{2} \mathrm{Cl}_{2}$ at $0{ }^{\circ} \mathrm{C}$ to give $57 \mathrm{mg}$ (57\% yield of crystals, $41 \%$ overall yield) of enantiomerically enriched product ( $85 \%$ ee).

tert-Butyl $(2 S, 4 S, 5 R)$-2-methoxycarbonyl-5-(2-furyl)pyrrolidine-4-carboxylate $(3 \mathrm{~m})$.

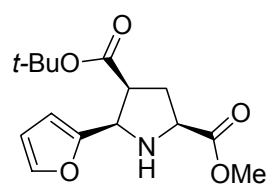

Obtained after purification by column chromatography using hexane-EtOAc (60:40) in $86 \%$ yield and $73 \%$ ee (Chiralpack AS, hexane/2-propanol 90:10, flow rate $1 \mathrm{~mL} / \mathrm{min}, \mathrm{t}_{\mathrm{R}(\text { major })}=5.8 \mathrm{~min}$, $\left.\mathrm{t}_{\mathrm{R}(\text { minor })}=9.7 \mathrm{~min}\right) ;[\alpha]_{\mathrm{D}}^{25}-9\left(\mathrm{c} 1.5, \mathrm{CH}_{2} \mathrm{Cl}_{2}\right) ; \mathrm{HRMS} \mathrm{C}_{15} \mathrm{H}_{21} \mathrm{NO}_{5}$

$[\mathrm{M}+\mathrm{Na}]^{+}$calculated 318.1312 , found $318.1318 ;{ }^{1} \mathrm{H}$ NMR $\left(400 \mathrm{MHz}, \mathrm{CDCl}_{3}\right) \delta 7.28$ $(1 \mathrm{H}, \mathrm{d}, J=2.0 \mathrm{~Hz}), 6.26(1 \mathrm{H}, \mathrm{dd}, J=3.2,2.0 \mathrm{~Hz}), 6.22(1 \mathrm{H}, \mathrm{d}, J=3.2 \mathrm{~Hz}), 4.46(1 \mathrm{H}, \mathrm{d}$, $J=8.0 \mathrm{~Hz}), 3.87(1 \mathrm{H}, \mathrm{t}, J=8.0 \mathrm{~Hz}), 3.73(3 \mathrm{H}, \mathrm{s}), 3.19(1 \mathrm{H}, \mathrm{q}, J=8.0 \mathrm{~Hz}), 2.65(1 \mathrm{H}, \mathrm{br}$ 
s), $2.35(2 \mathrm{H}, \mathrm{t}, J=8.0 \mathrm{~Hz}), 1.20(9 \mathrm{H}, \mathrm{s}) ;{ }^{13} \mathrm{C} \mathrm{NMR}\left(100 \mathrm{MHz}, \mathrm{CDCl}_{3}\right) \delta 173.8,170.8$, $153.4,141.5,110.1,107.0,80.7,59.2,58.9,52.2,49.3,32.5,27.6(3 \mathrm{C})$.

tert-Butyl (2S, 4S, 5R)-2-methoxycarbonyl-5-(1-naphthyl)pyrrolidine-4-carboxylate (3n).

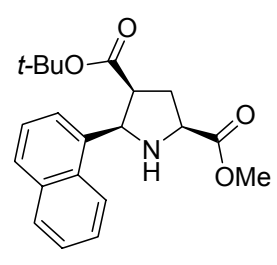

Obtained after purification by column chromatography using hexane-EtOAc (70:30) in $88 \%$ yield and $64 \%$ ee (Chiralpack AS, hexane/2-propanol 90:10, flow rate $1 \mathrm{~mL} / \mathrm{min}, \mathrm{t}_{\mathrm{R}(\text { major })}=7.6 \mathrm{~min}$, $\left.\mathrm{t}_{\mathrm{R}(\text { minor })}=17.1 \mathrm{~min}\right) . \quad[\alpha]_{\mathrm{D}}^{25}+126 \quad\left(c \quad 0.49, \mathrm{CH}_{2} \mathrm{Cl}_{2}\right) ; \quad$ HRMS $\mathrm{C}_{21} \mathrm{H}_{25} \mathrm{NO}_{4}[\mathrm{M}+\mathrm{Na}]^{+}$calculated 378.1676, found $378.1675 ;{ }^{1} \mathrm{H}$ NMR (400 MHz, $\left.\mathrm{CDCl}_{3}\right) \delta 7.99(1 \mathrm{H}, \mathrm{d}, J=8.0 \mathrm{~Hz}), 7.84(1 \mathrm{H}, \mathrm{d}, J=7.6 \mathrm{~Hz}), 7.75(1 \mathrm{H}$, $\mathrm{d}, J=8.0 \mathrm{~Hz}), 7.67(1 \mathrm{H}, \mathrm{d}, J=7.6 \mathrm{~Hz}), 7.51(1 \mathrm{H}, \mathrm{t}, J=7.6 \mathrm{~Hz}), 7.46(1 \mathrm{H}, \mathrm{t}, J=8 \mathrm{~Hz})$, $7.43(1 \mathrm{H}, \mathrm{t}, J=8.0 \mathrm{~Hz}), 5.13(1 \mathrm{H}, \mathrm{dd}, J=8.0 \mathrm{~Hz}), 3.82(3 \mathrm{H}, \mathrm{s}), 3.52(1 \mathrm{H}, \mathrm{dd}, J=8.0$, $13.6 \mathrm{~Hz}), 2.91\left(1 \mathrm{H}\right.$, br s), 2.55-2.40 (2H, m), $0.60(9 \mathrm{H}, \mathrm{s}) ;{ }^{13} \mathrm{C} \mathrm{NMR}\left(100 \mathrm{MHz}, \mathrm{CDCl}_{3}\right)$ $\delta 173.3,171.7,134.4,133.3,131.4,128.5,127.8,125.7,125.3(2 \mathrm{C}), 123.4(2 \mathrm{C}), 79.8$, $62.0,59.4,52.1,48.7,33.7,26.9(3 \mathrm{C})$.

tert-Butyl (2S, $4 S, 5 R)$-2-methoxycarbonyl-5-(2-naphthyl)pyrrolidine-4-carboxylate (3o).

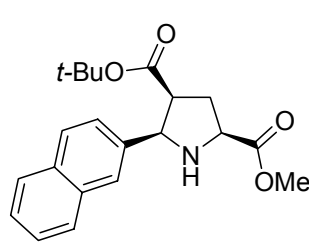

Obtained after purification by column chromatography using $\mathrm{CH}_{2} \mathrm{Cl}_{2}-\mathrm{Et}_{2} \mathrm{O}$ (80:20) in $86 \%$ yield and $62 \%$ ee (Chiralpack AS, hexane/2-propanol 95:5, flow rate $1 \mathrm{~mL} / \mathrm{min}, \mathrm{t}_{\mathrm{R}(\text { major })}=8.9 \mathrm{~min}$, $\left.\mathrm{t}_{\mathrm{R}(\text { minor })}=16.8 \mathrm{~min}\right) .[\alpha]^{25}+17\left(c 0.45, \mathrm{CH}_{2} \mathrm{Cl}_{2}\right) ;{ }^{1} \mathrm{H} \mathrm{NMR}$ and ${ }^{13} \mathrm{C}$ NMR were consistent with previously reported values for its enantiomer. $^{1}$

tert-Butyl $(2 S, 4 S, 5 S)$-2-methoxycarbonyl-5-(1-naphthyl)pyrrolidine-4-carboxylate (3p).

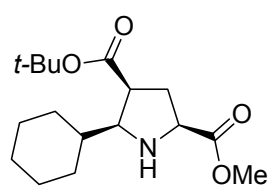

Obtained after purification by column chromatography using hexaneEtOAc (70:30) in 65\% yield and $41 \%$ ee $(2 \times$ Chiralpack AS, hexane/2-propanol 99:1, flow rate $0.5 \mathrm{~mL} / \mathrm{min}, \mathrm{t}_{\mathrm{R}(\text { major })}=21.8 \mathrm{~min}$, $\left.\mathrm{t}_{\mathrm{R}(\text { minor })}=26.4 \mathrm{~min}\right) .[\alpha]_{\mathrm{D}}^{25}-3\left(c \mathrm{c} 0.52, \mathrm{CH}_{2} \mathrm{Cl}_{2}\right)$; HRMS $\mathrm{C}_{17} \mathrm{H}_{29} \mathrm{NO}_{4}$ $[\mathrm{M}+\mathrm{Na}]^{+}$calculated 334.1989, found 339.1996; ${ }^{1} \mathrm{H} \mathrm{NMR}\left(400 \mathrm{MHz}, \mathrm{CDCl}_{3}\right) \delta 3.77$ 
$(1 \mathrm{H}, \mathrm{dd}, J=10.4,6.0 \mathrm{~Hz}), 3.73(3 \mathrm{H}, \mathrm{s}), 2.81(1 \mathrm{H}, \mathrm{td}, J=6.0,2.0 \mathrm{~Hz}), 2.73(1 \mathrm{H}, \mathrm{dd}, J=$ 10.0, $6.0 \mathrm{~Hz}), 2.32-2.24(2 \mathrm{H}, \mathrm{m}), 2.11(1 \mathrm{H}, \mathrm{ddd}, J=13.6,5.6,2.0 \mathrm{~Hz}), 2.06(1 \mathrm{H}, \mathrm{br} \mathrm{d}, J$ $=12.8 \mathrm{~Hz}), 1.97(1 \mathrm{H}$, br d, $J=12.8 \mathrm{~Hz}), 1.75-1.61(3 \mathrm{H}, \mathrm{m}), 1.41(9 \mathrm{H}, \mathrm{s}), 1.40-0.95(6 \mathrm{H}$, $\mathrm{m}) ;{ }^{13} \mathrm{C} \mathrm{NMR}\left(100 \mathrm{MHz}, \mathrm{CDCl}_{3}\right) \delta 173.9,173.6,80.7,70.1,59.6,52.0,46.5,39.4,34.9$, $31.8,31.0,28.0(3 \mathrm{C}), 26.2,25.9,25.8$.

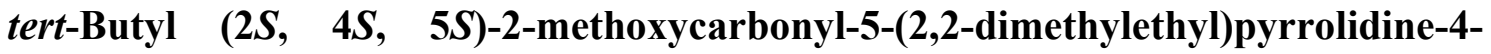 carboxylate (3q).}

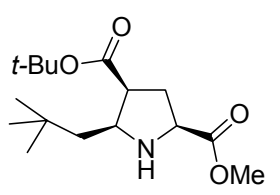

Obtained after purification by column chromatography using hexaneEtOAc $(65: 35)$ in $78 \%$ yield and $41 \%$ ee $(2 \times$ Chiralpack AS, hexane/2-propanol 99:1, flow rate $0.3 \mathrm{~mL} / \mathrm{min}, \mathrm{t}_{\mathrm{R} \text { (major) }}=29.4 \mathrm{~min}$, $\left.\mathrm{t}_{\mathrm{R}(\text { minor })}=32.5 \mathrm{~min}\right) .[\alpha]_{\mathrm{D}}^{25}-14\left(c \quad 0.87, \mathrm{CH}_{2} \mathrm{Cl}_{2}\right)$; HRMS $\mathrm{C}_{16} \mathrm{H}_{29} \mathrm{NO}_{4}$ $[\mathrm{M}+\mathrm{Na}]^{+}$calculated 322.1989 , found $322.1996 ;{ }^{1} \mathrm{H} \mathrm{NMR}\left(400 \mathrm{MHz}, \mathrm{CDCl}_{3}\right) \delta 3.73$ $(1 \mathrm{H}, \mathrm{dd}, J=9.6,7.2 \mathrm{~Hz}), 3.71(3 \mathrm{H}, \mathrm{s}), 3.11(1 \mathrm{H}, \mathrm{dd}, J=10.8,7.2 \mathrm{~Hz}), 2.73(1 \mathrm{H}, \mathrm{td}, J=$ 8.4, 3.6 Hz), 2.29-2.21 (2H, m), $2.07(1 \mathrm{H}, \mathrm{ddd}, J=13.6,6.4,3.2 \mathrm{~Hz}), 1.56(1 \mathrm{H}, \mathrm{dd}, J=$ 14.4, $3.6 \mathrm{~Hz}), 1.40(9 \mathrm{H}, \mathrm{s}), 1.43-1.29(1 \mathrm{H}, \mathrm{m}), 0.92(9 \mathrm{H}, \mathrm{s}) ;{ }^{13} \mathrm{C} \mathrm{NMR}(100 \mathrm{MHz}$, $\left.\mathrm{CDCl}_{3}\right) \delta 173.8,173.5,80.8,60.5,59.9,52.0,49.6,44.3,34.3,30.2,29.8(3 \mathrm{C}), 28.1$ $(3 \mathrm{C})$.

tert-Butyl $(2 S, 4 S, 5 S)$-2-methoxycarbonyl-5-isopropylpyrrolidine-4-carboxylate (3r).

Obtained after purification by column chromatography using hexane-

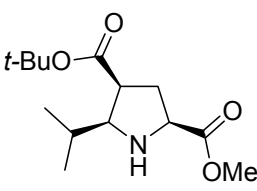
EtOAc (70:30) in 67\% yield and 52\% ee ( 2 x Chiralpack AS, hexane/2propanol 99:1, flow rate $0.3 \mathrm{~mL} / \mathrm{min}, \mathrm{t}_{\mathrm{R}(\text { major })}=31.3 \mathrm{~min}, \mathrm{t}_{\mathrm{R}(\text { minor })}=38.6$ $\min ) .[\alpha]^{25}-8\left(c\right.$ 1.1, $\left.\mathrm{CH}_{2} \mathrm{Cl}_{2}\right)$; HRMS $\mathrm{C}_{14} \mathrm{H}_{25} \mathrm{NO}_{4}[\mathrm{M}+\mathrm{Na}]^{+}$calculated 294.1676, found 294.1681; ${ }^{1} \mathrm{H}$ NMR (400 MHz, $\left.\mathrm{CDCl}_{3}\right) \delta 3.81(1 \mathrm{H}, \mathrm{dd}$, $J=10.4,5.6 \mathrm{~Hz}), 3.75(3 \mathrm{H}, \mathrm{s}), 2.82(1 \mathrm{H}, \mathrm{td}, J=6.6,2.0 \mathrm{~Hz}), 2.67(1 \mathrm{H}, \mathrm{dd}, J=10.4,6.0$ Hz), 2.36-2.28 (1H, m), 2.14 (1H, ddd, $J=13.6,6.6,2.0 \mathrm{~Hz}), 1.71-1.41(1 \mathrm{H}, \mathrm{m}), 1.42$ $(9 \mathrm{H}, \mathrm{s}), 1.10(3 \mathrm{H}, \mathrm{d}, J=6.4 \mathrm{~Hz}), 1.02(3 \mathrm{H}, \mathrm{d}, J=6.4 \mathrm{~Hz}) ;{ }^{13} \mathrm{C} \mathrm{NMR}\left(100 \mathrm{MHz}, \mathrm{CDCl}_{3}\right)$ $\delta 173.7,173.5,80.7,71.6,59.7,52.0,46.9,35.1,29.9,28.0$ (3C), 21.8, 20.8. 
General procedure for the racemic 1,3 dipolar cycloaddition. The same procedure for the asymmetric reaction was carried out, using $\mathrm{Et}_{3} \mathrm{~N}$ instead of hydrocinchonine, at room temperature overnight. The reaction mixture was filtered through a pad of silica gel eluting with diethyl ether. Racemic products were used without further purification for comparing enantiomer retention times in HPLC.

(1) ) Chen, C.; Li, X.; Schreiber, S. L. J. Am. Chem. Soc. 2003, 125, 10174-10175. 


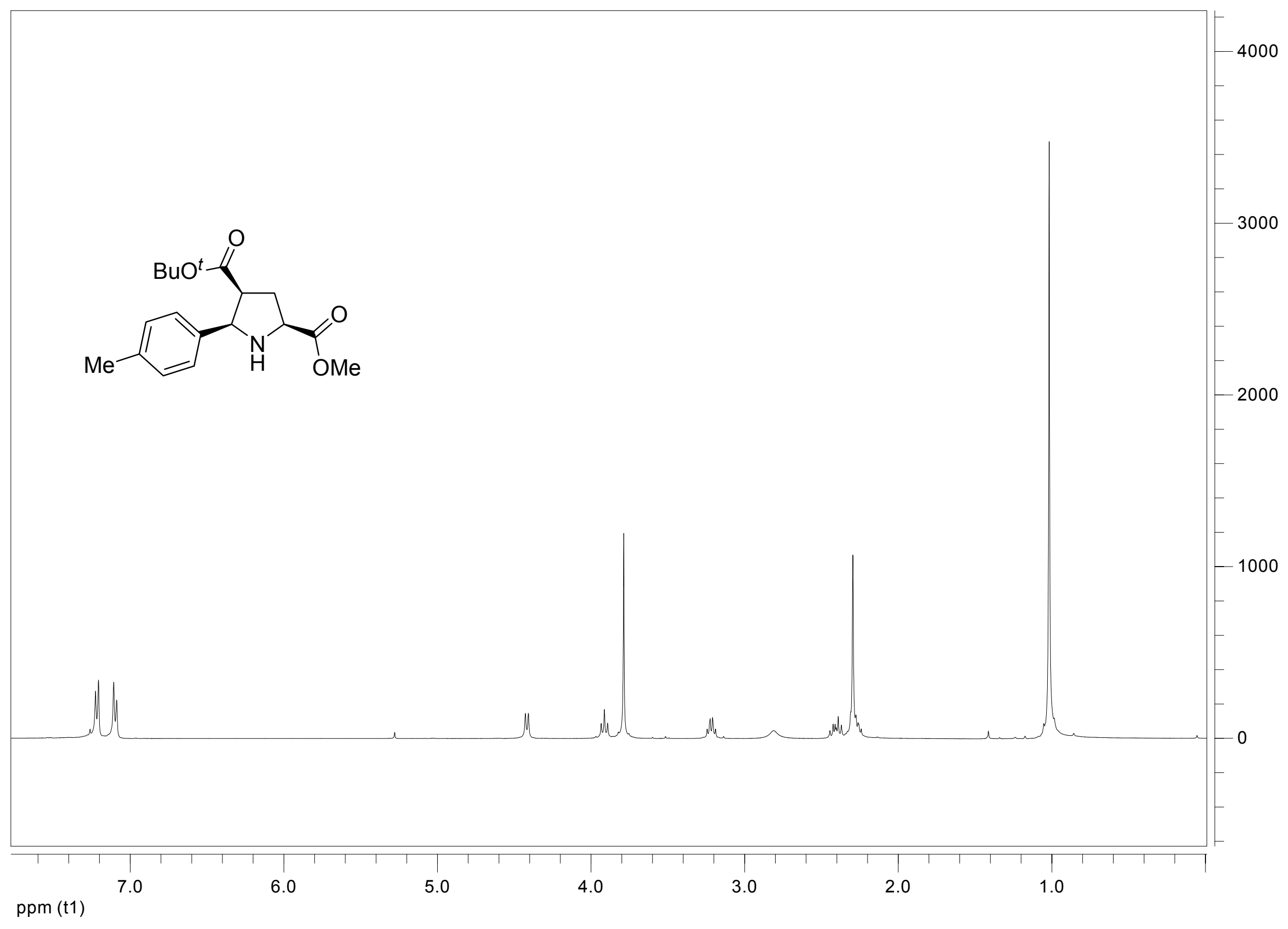




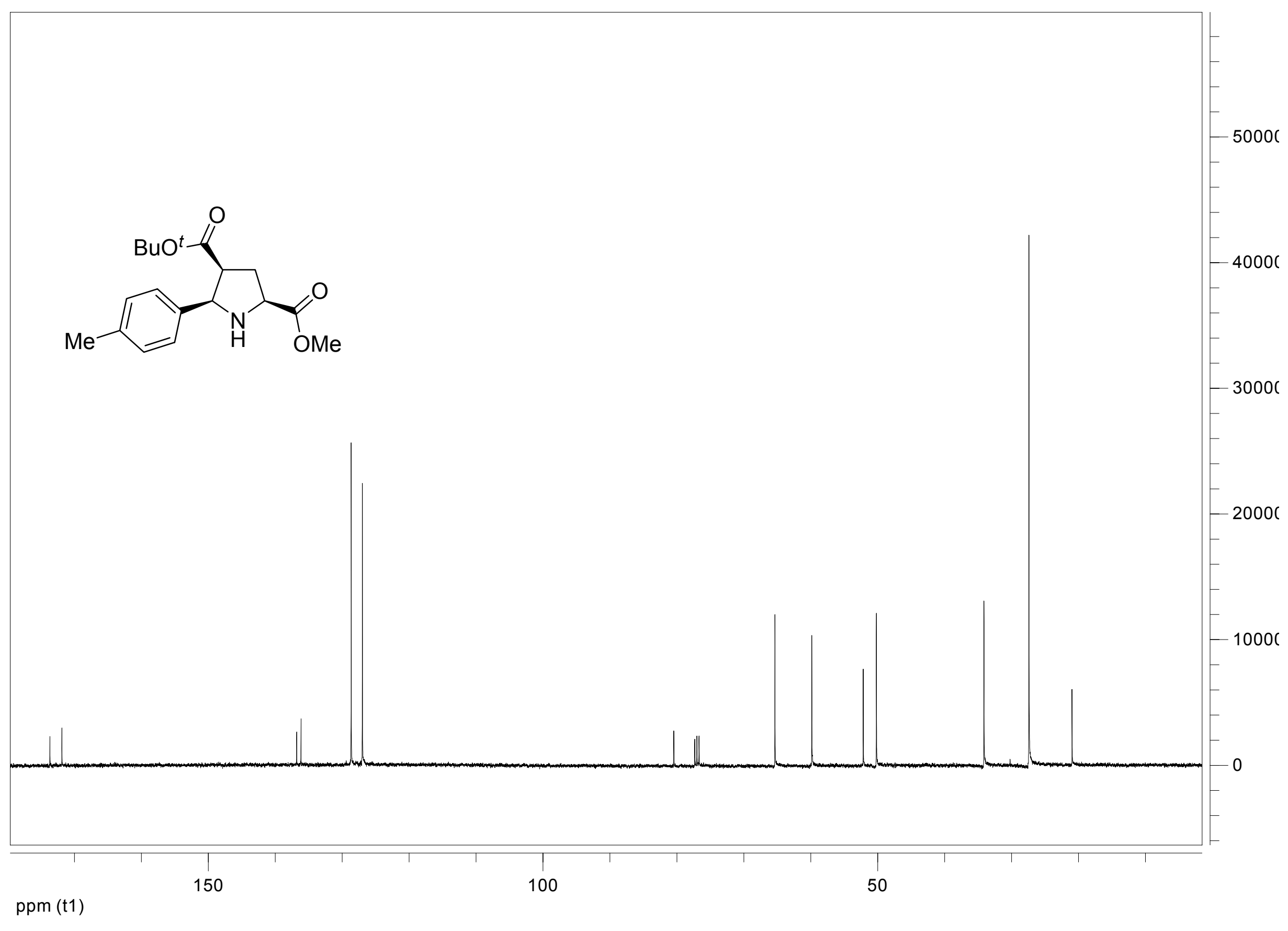




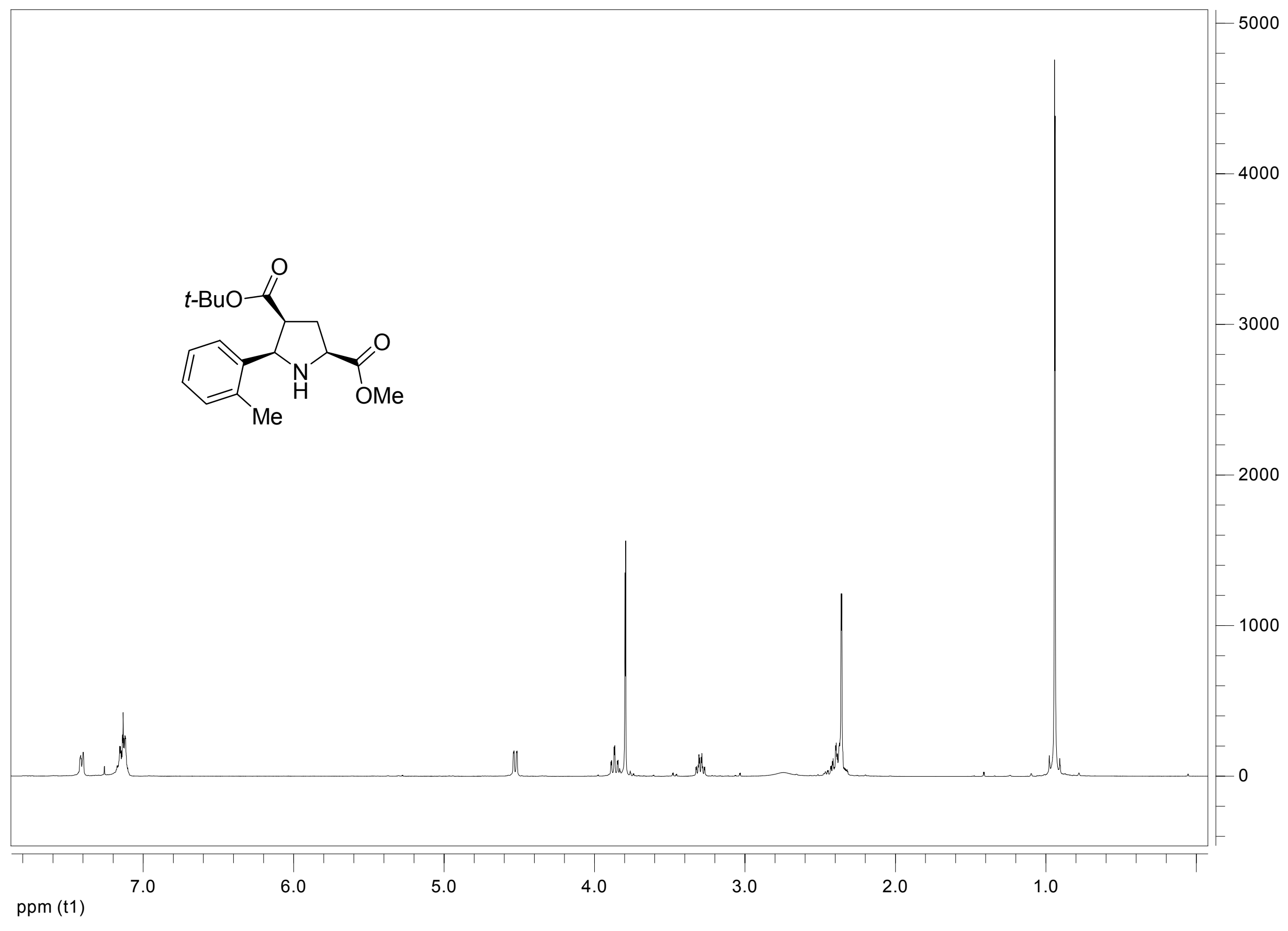




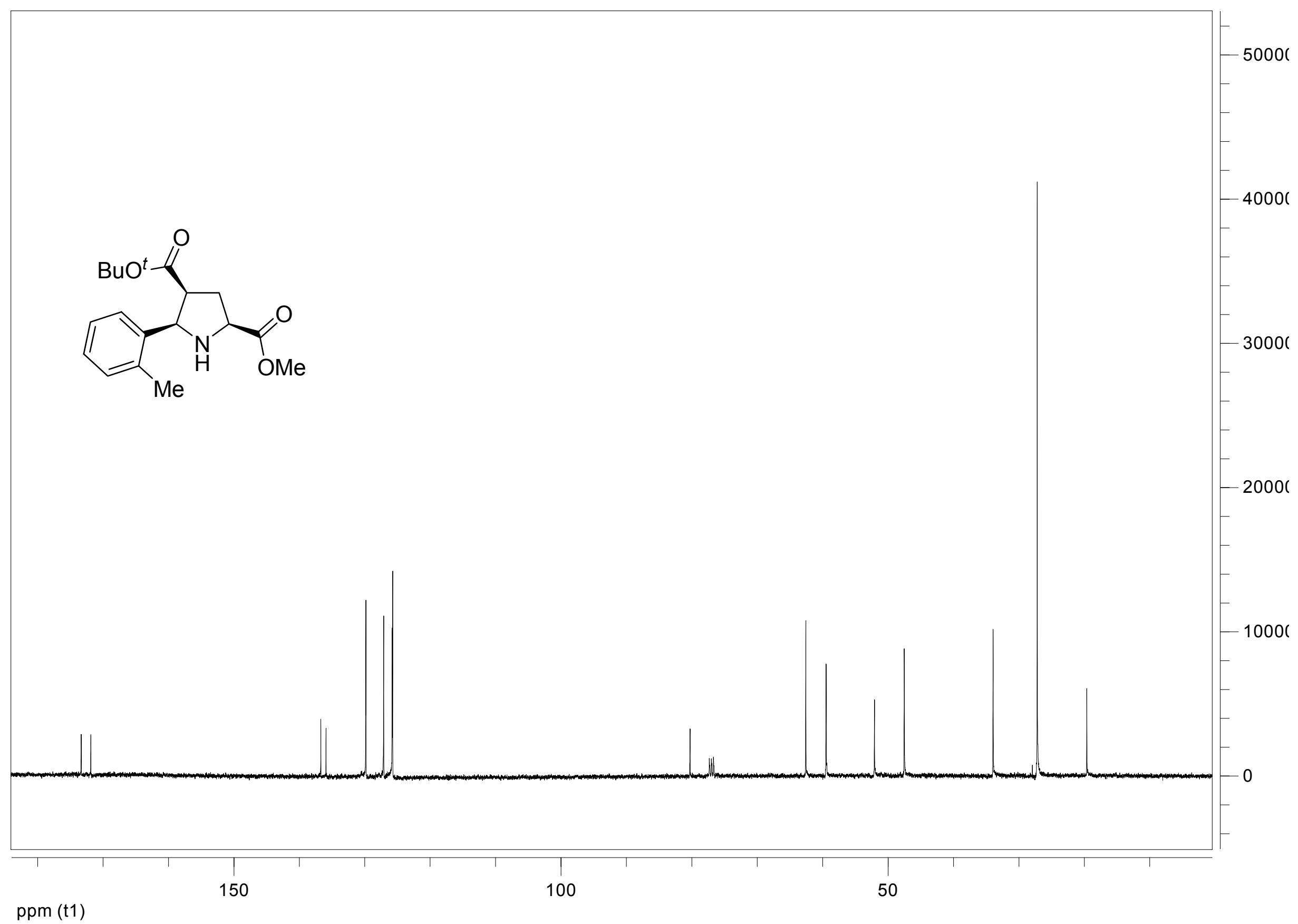




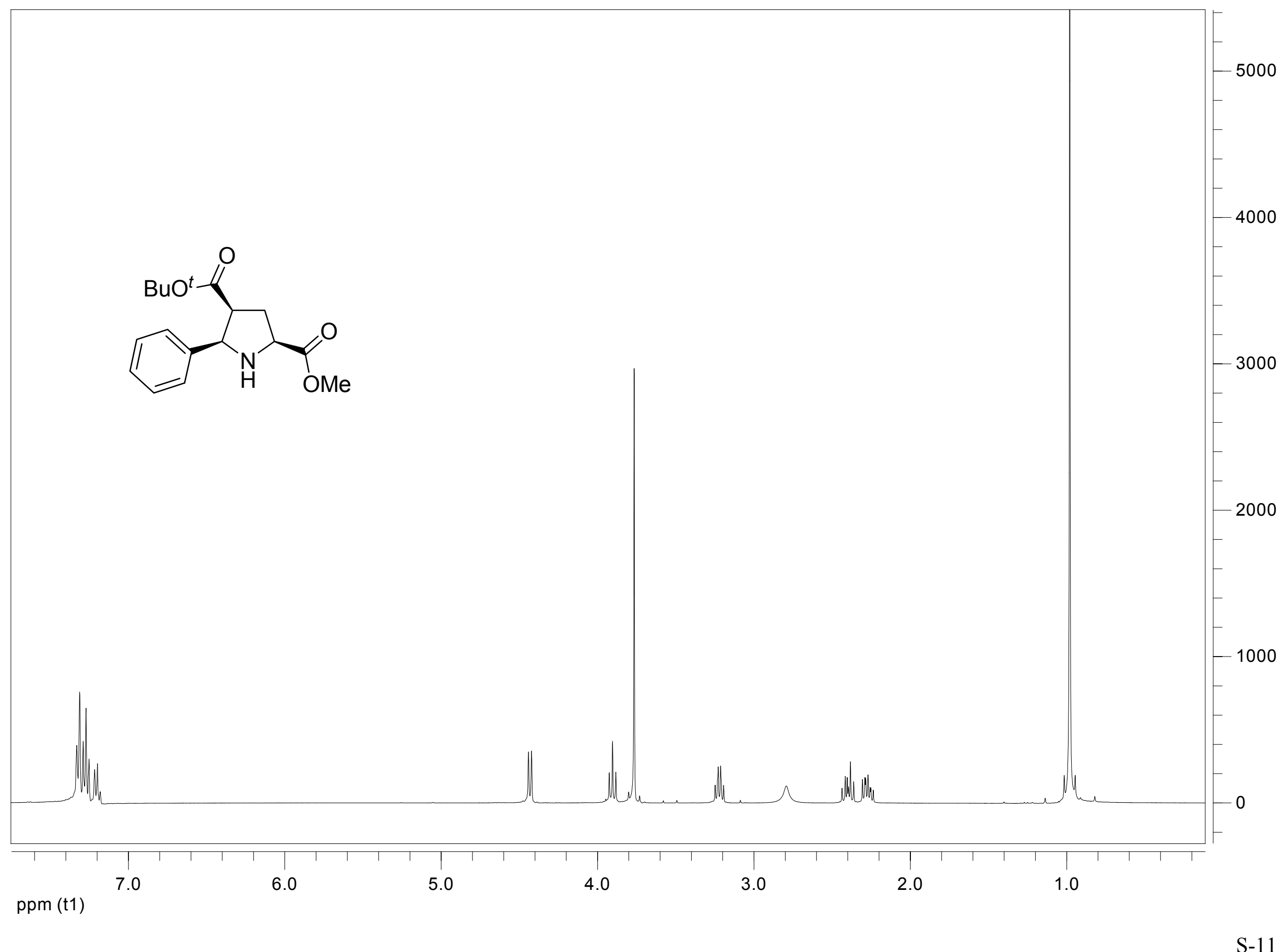




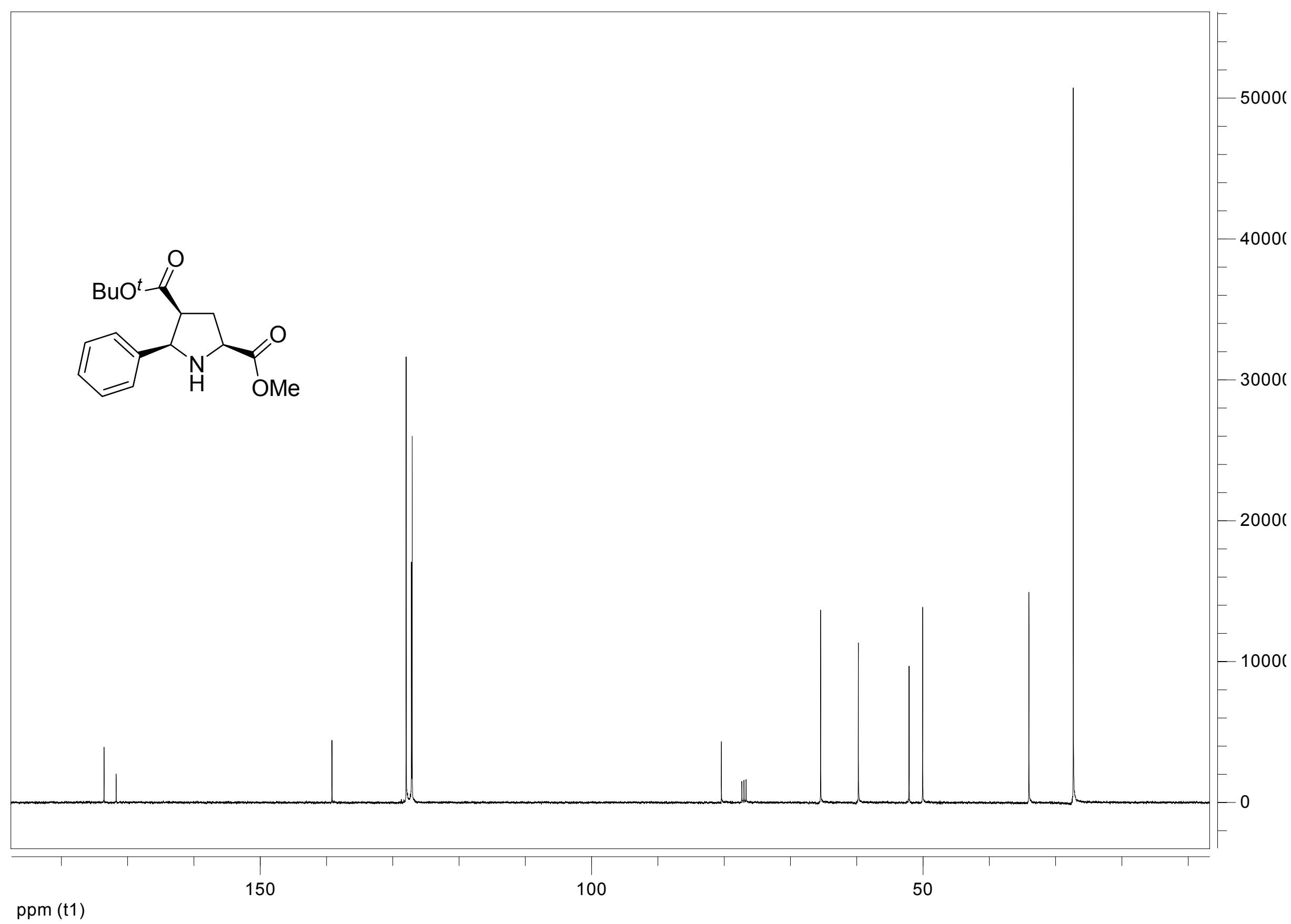




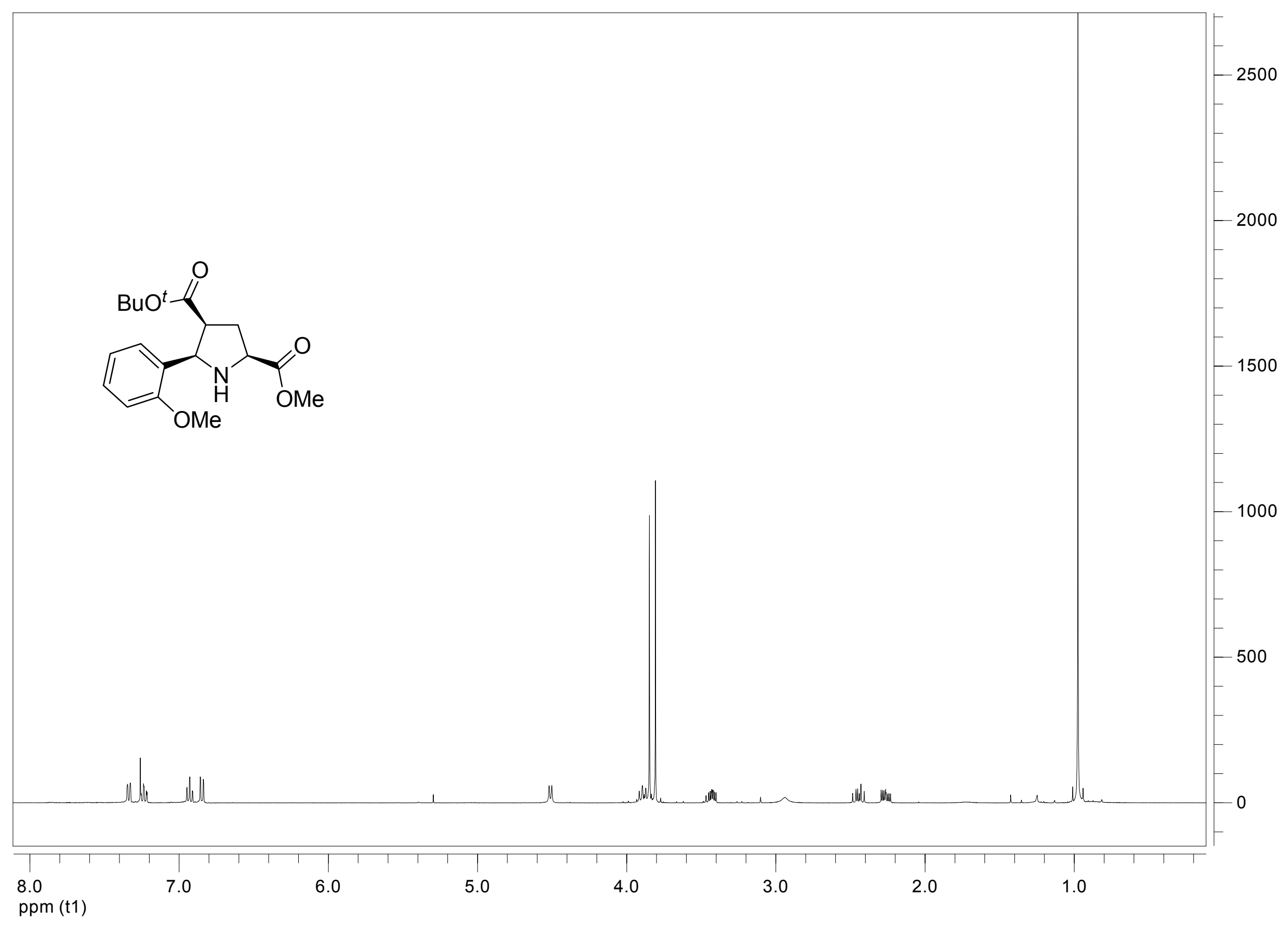




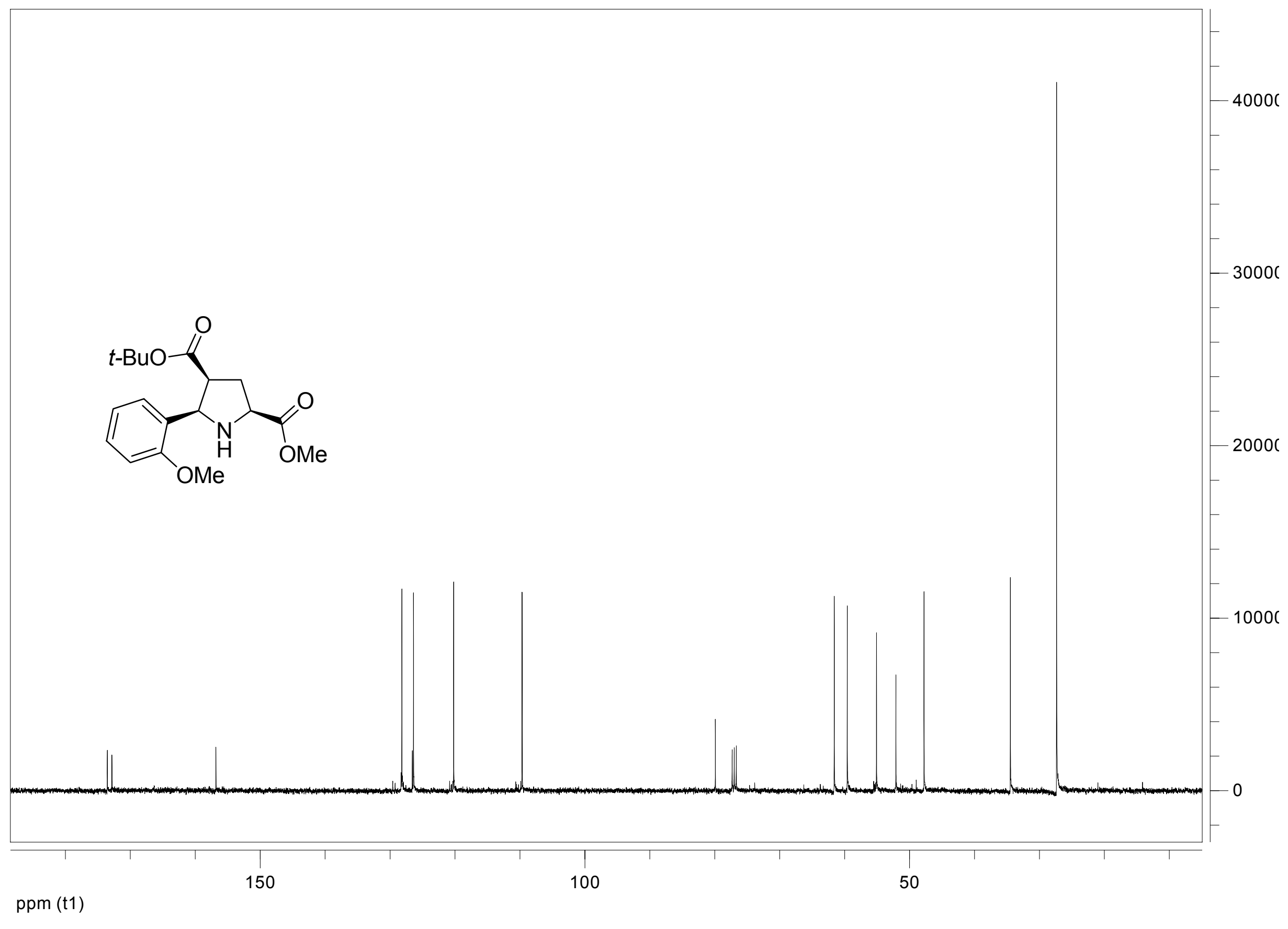




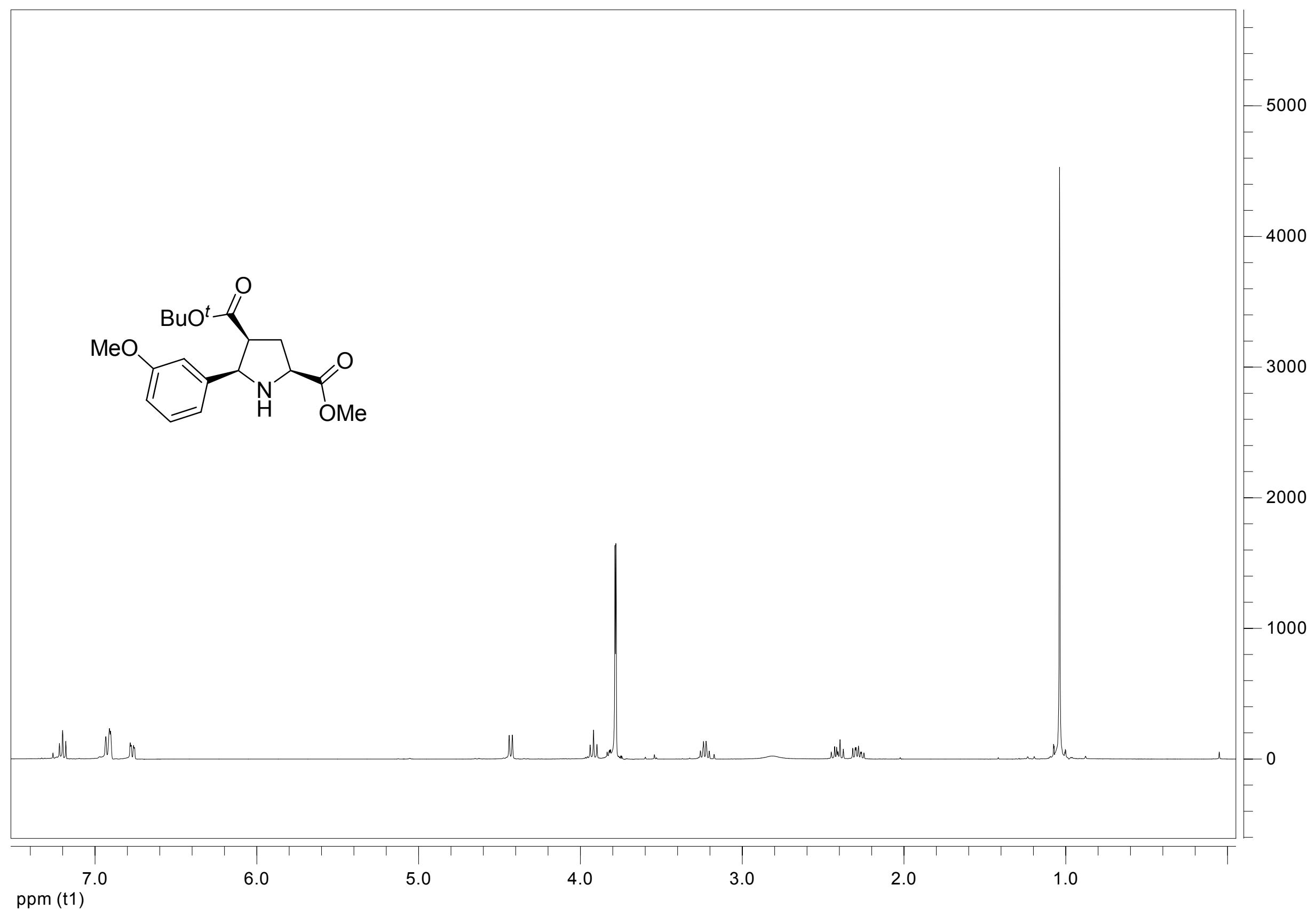




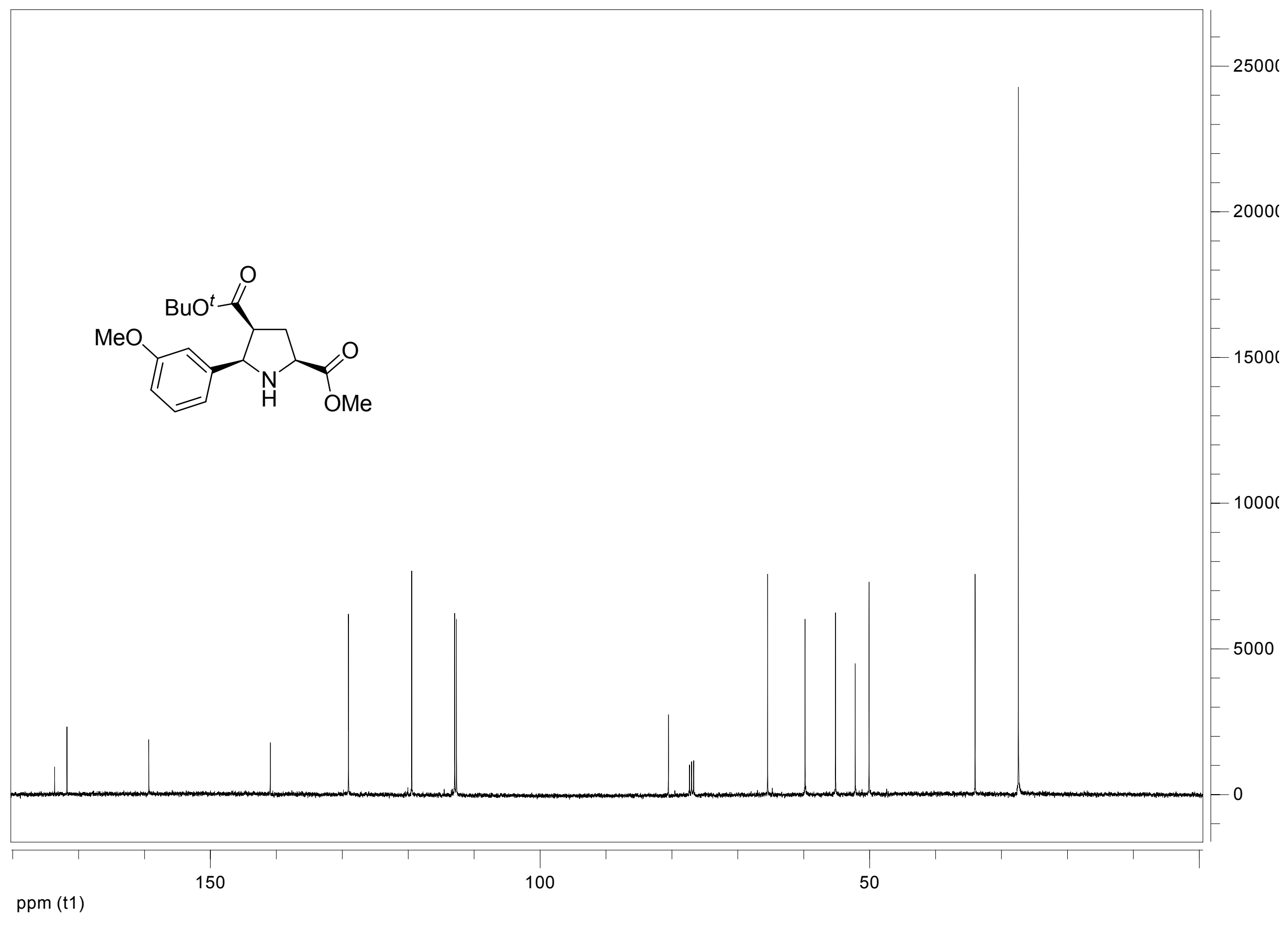




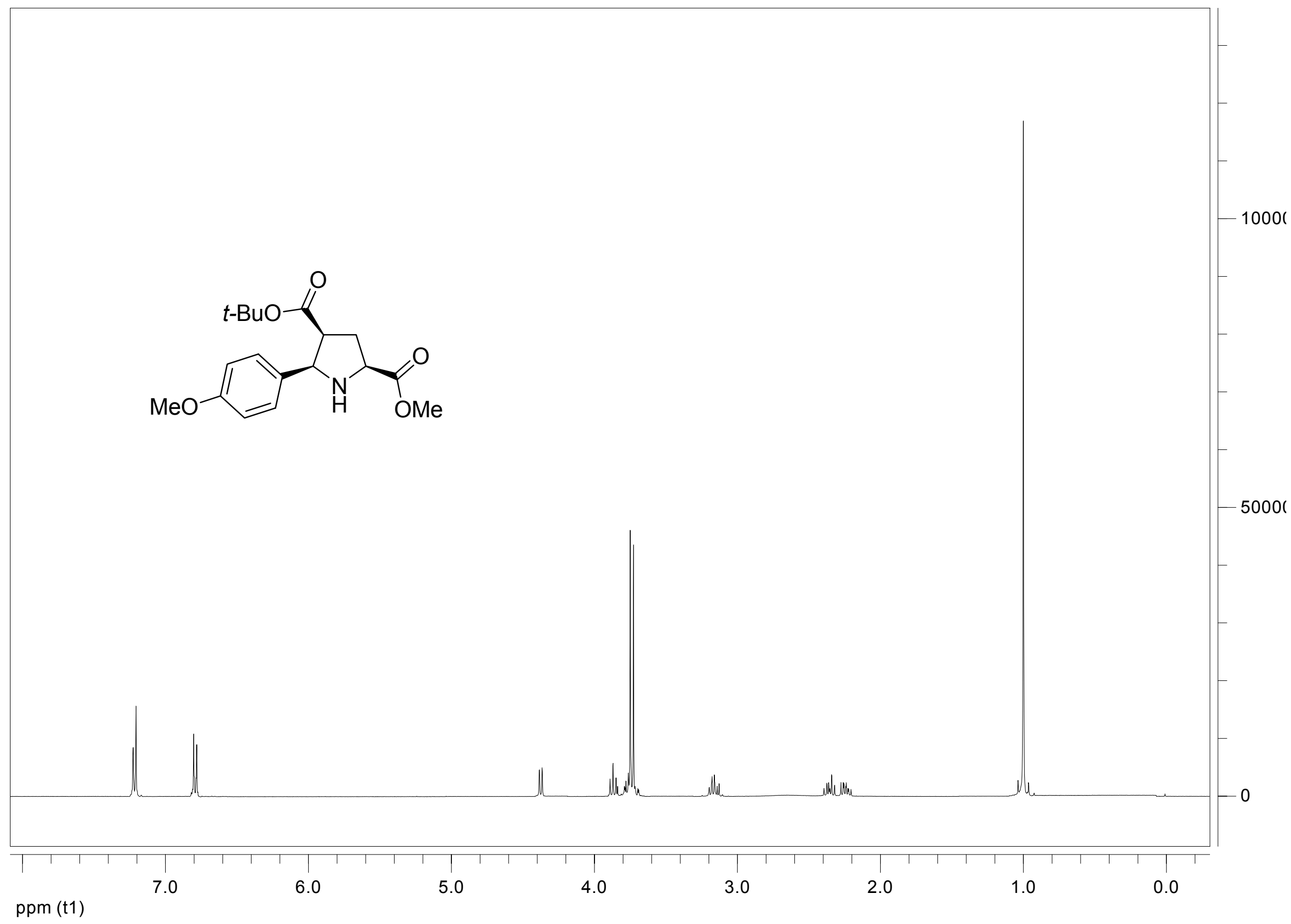




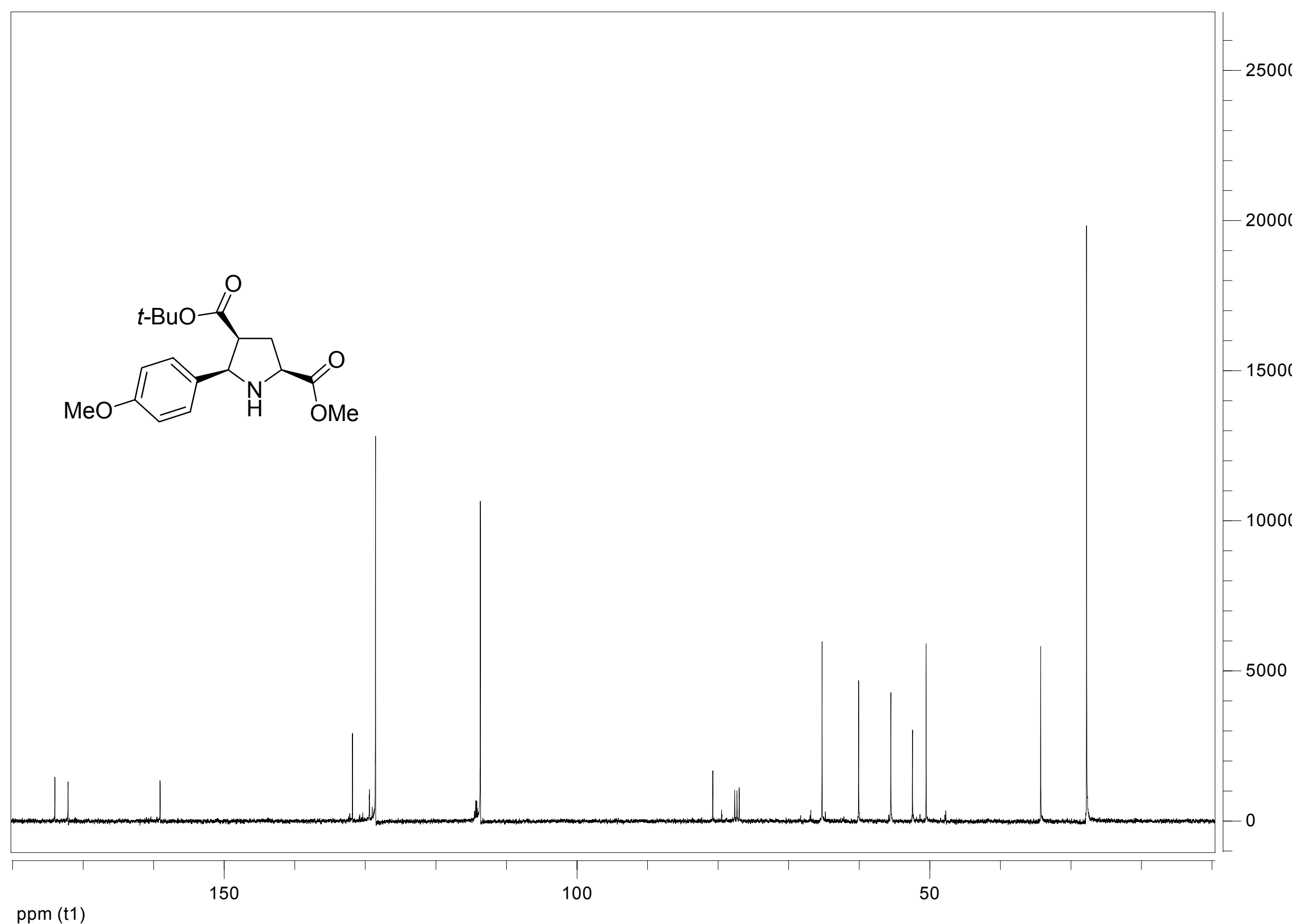




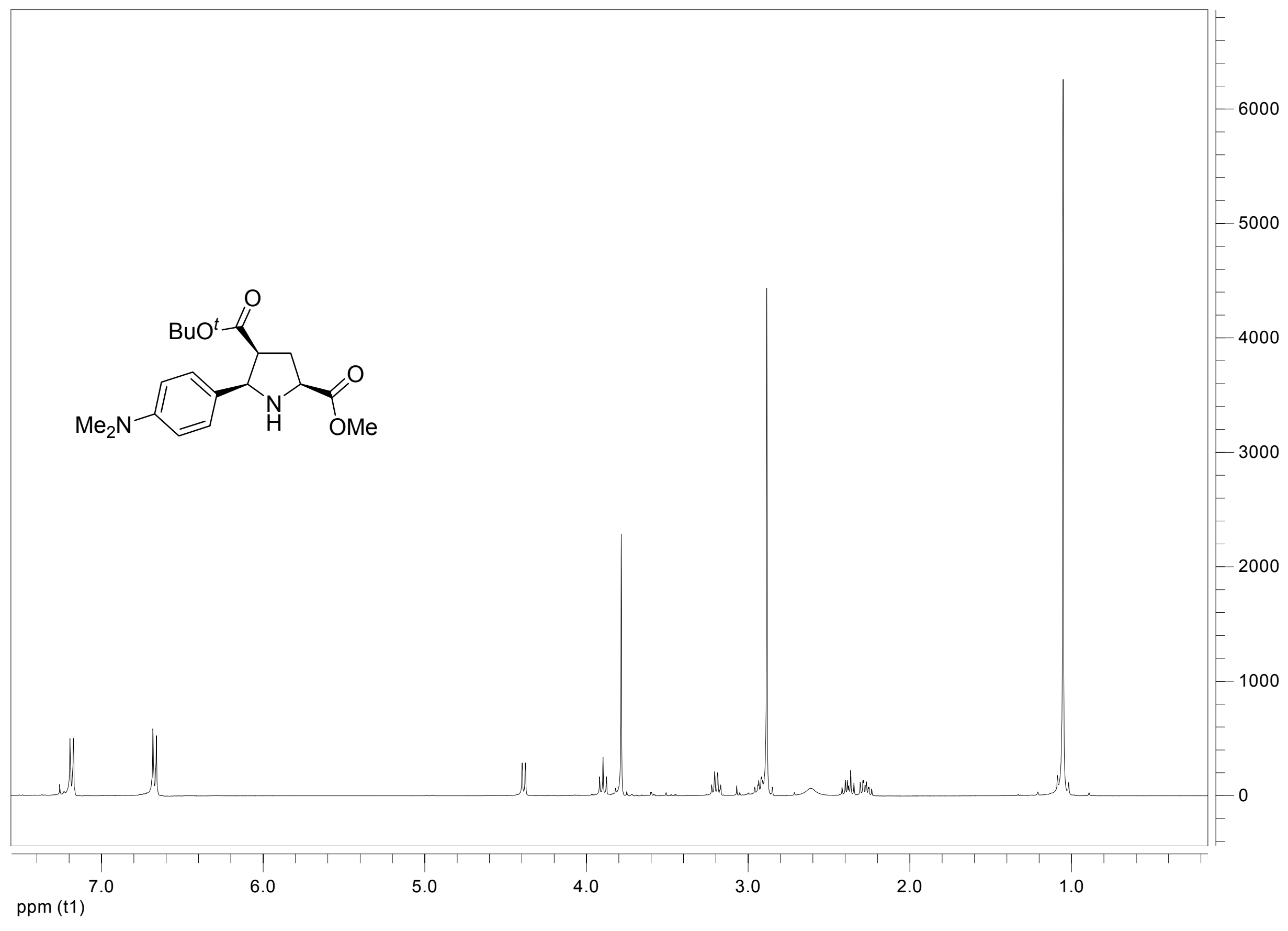




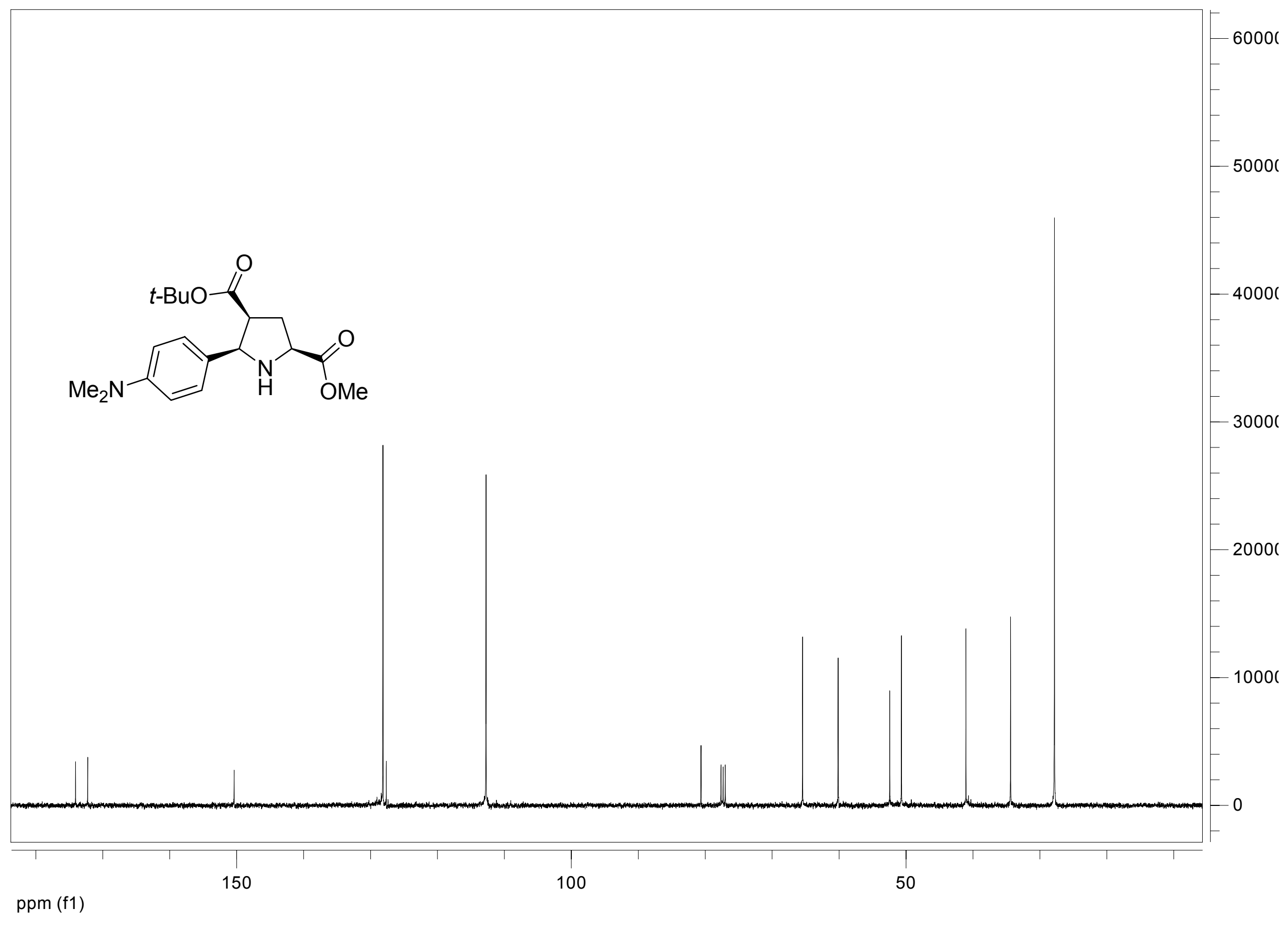




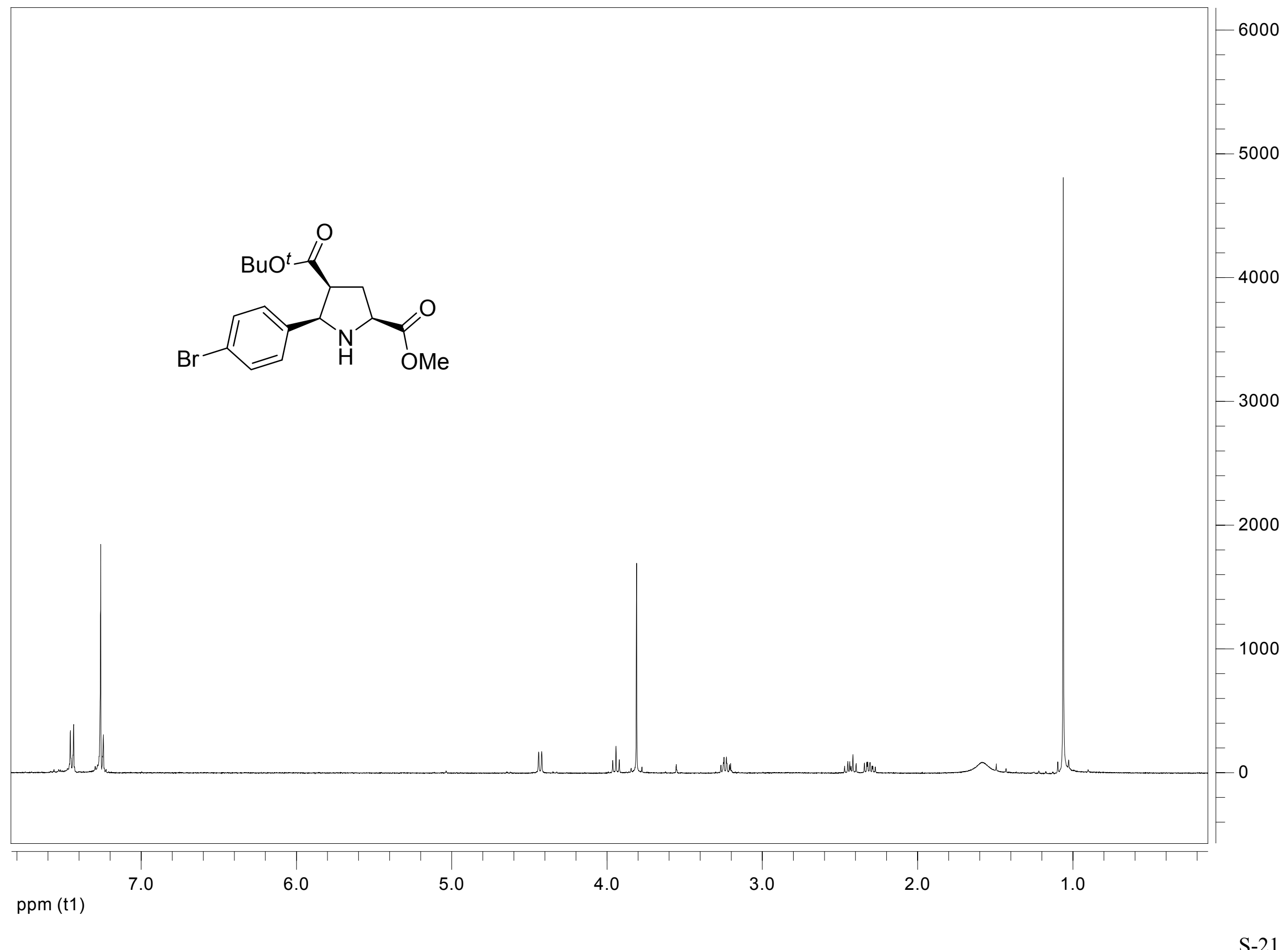




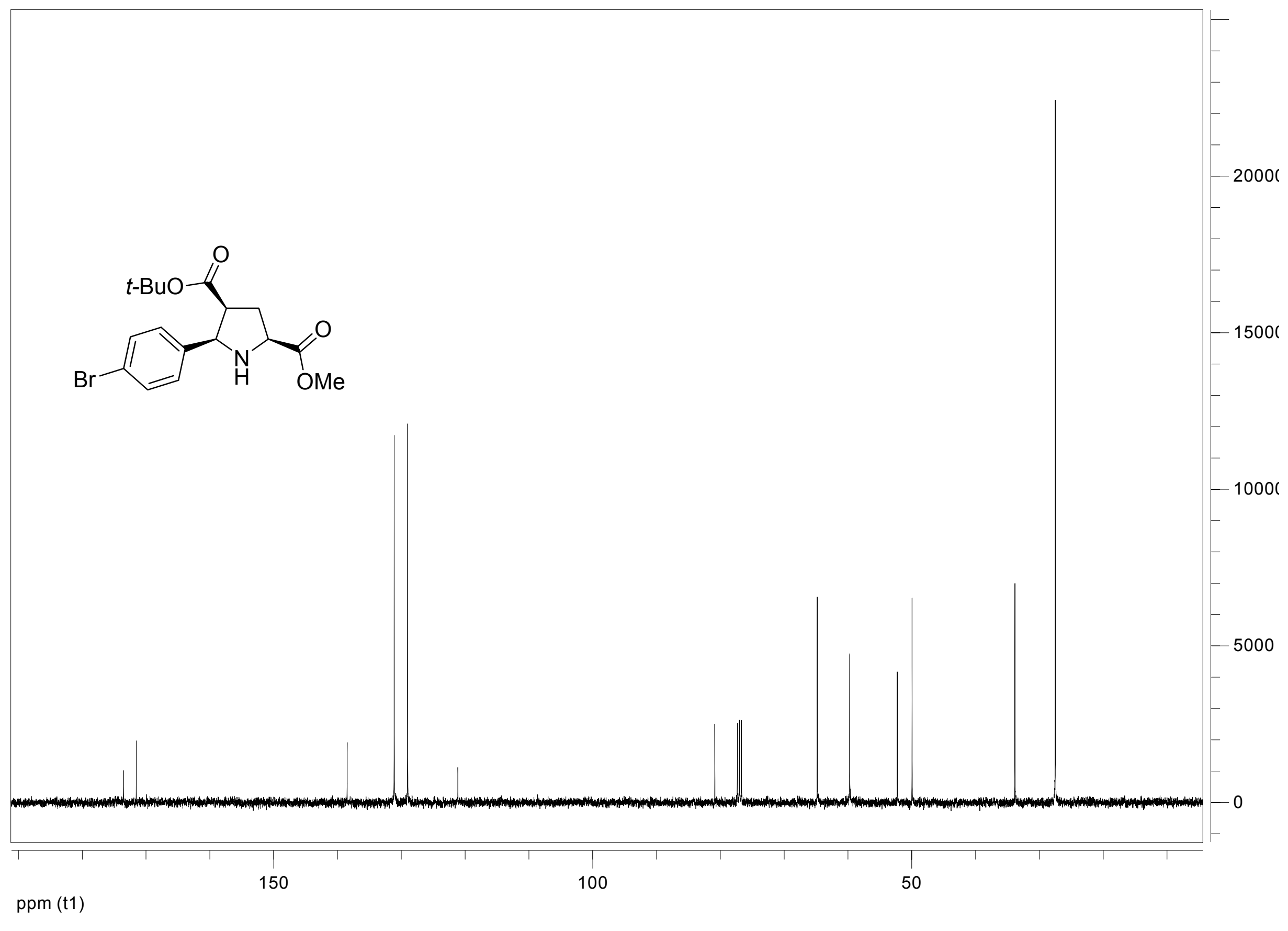




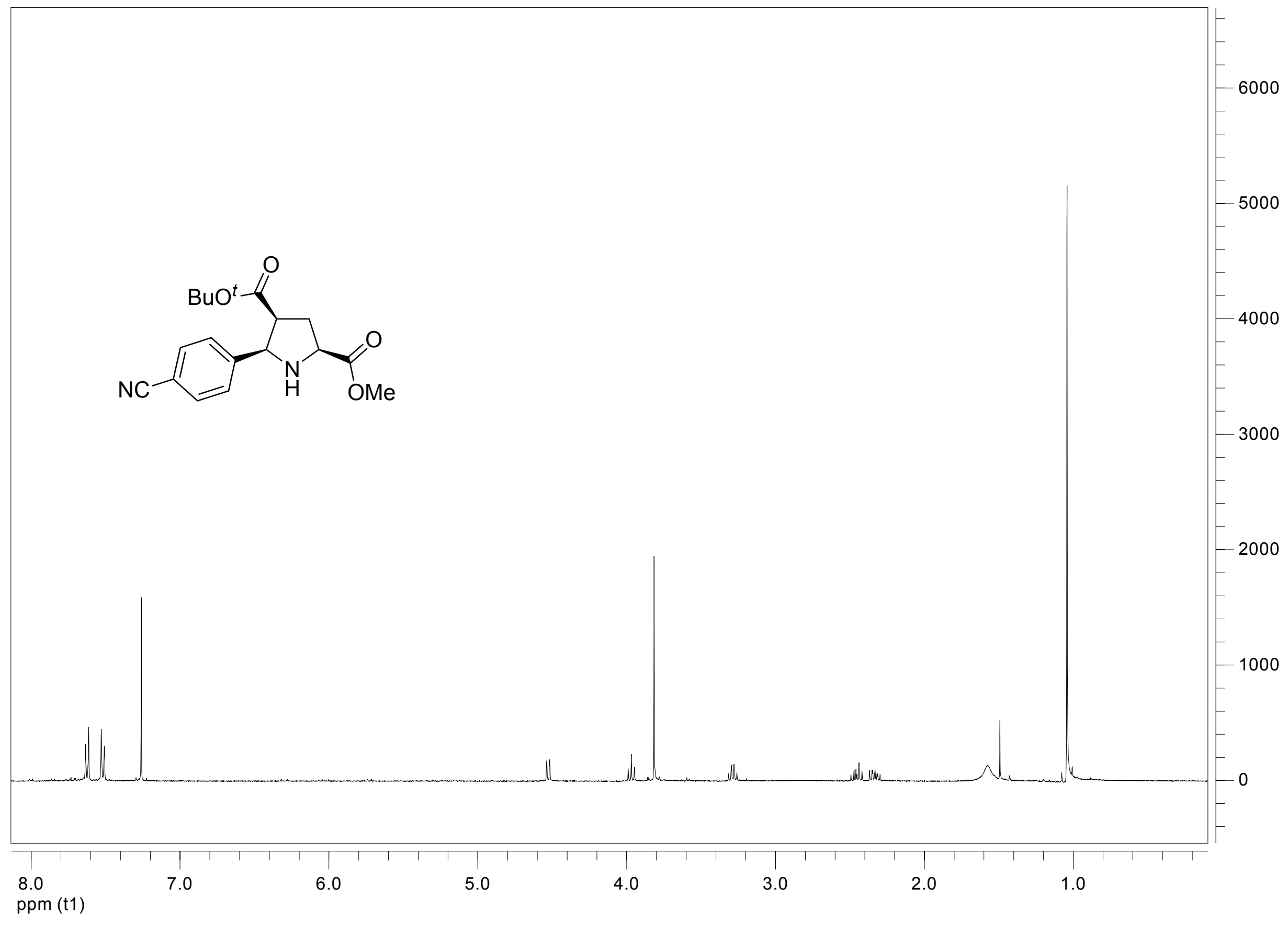




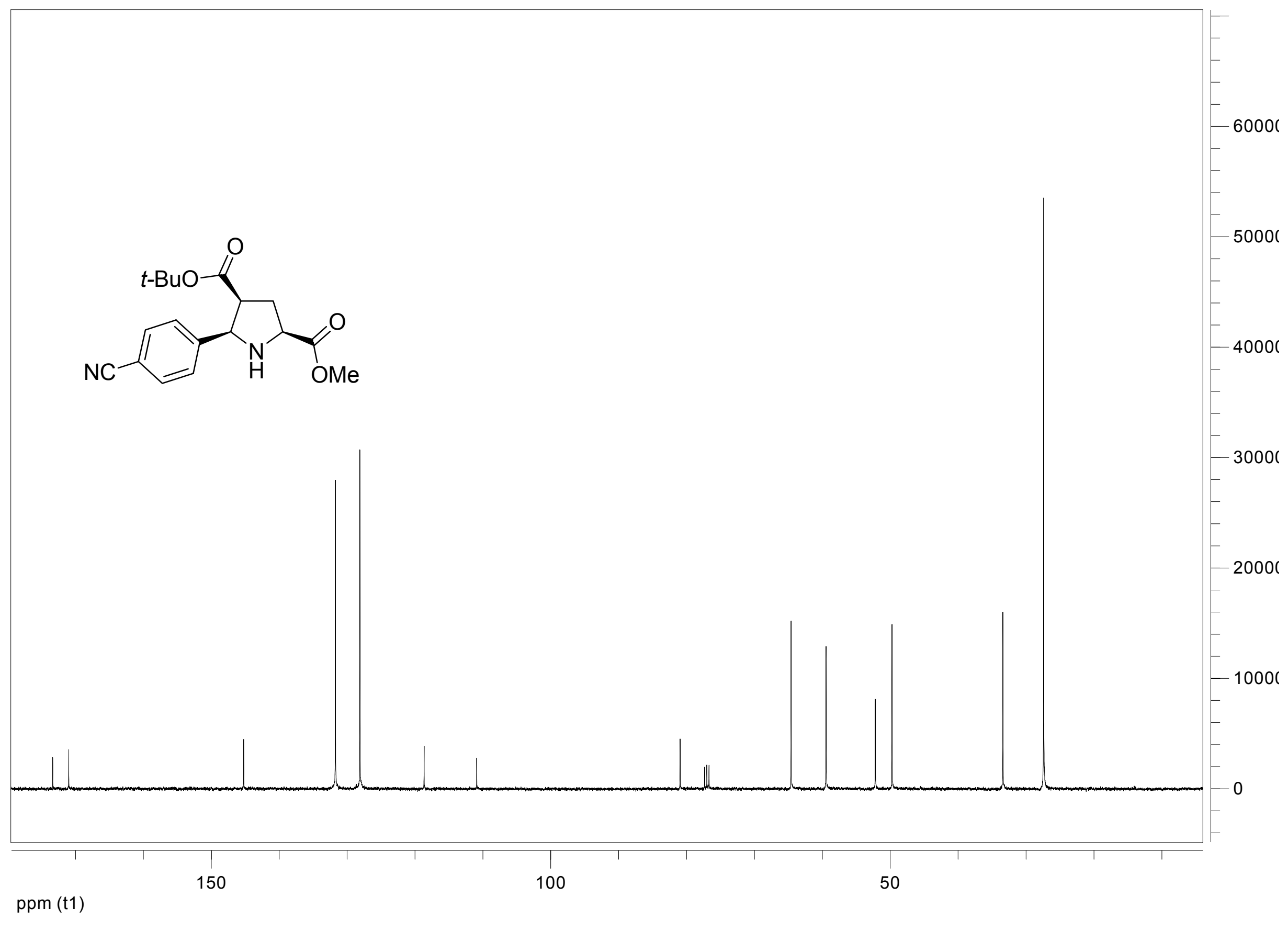




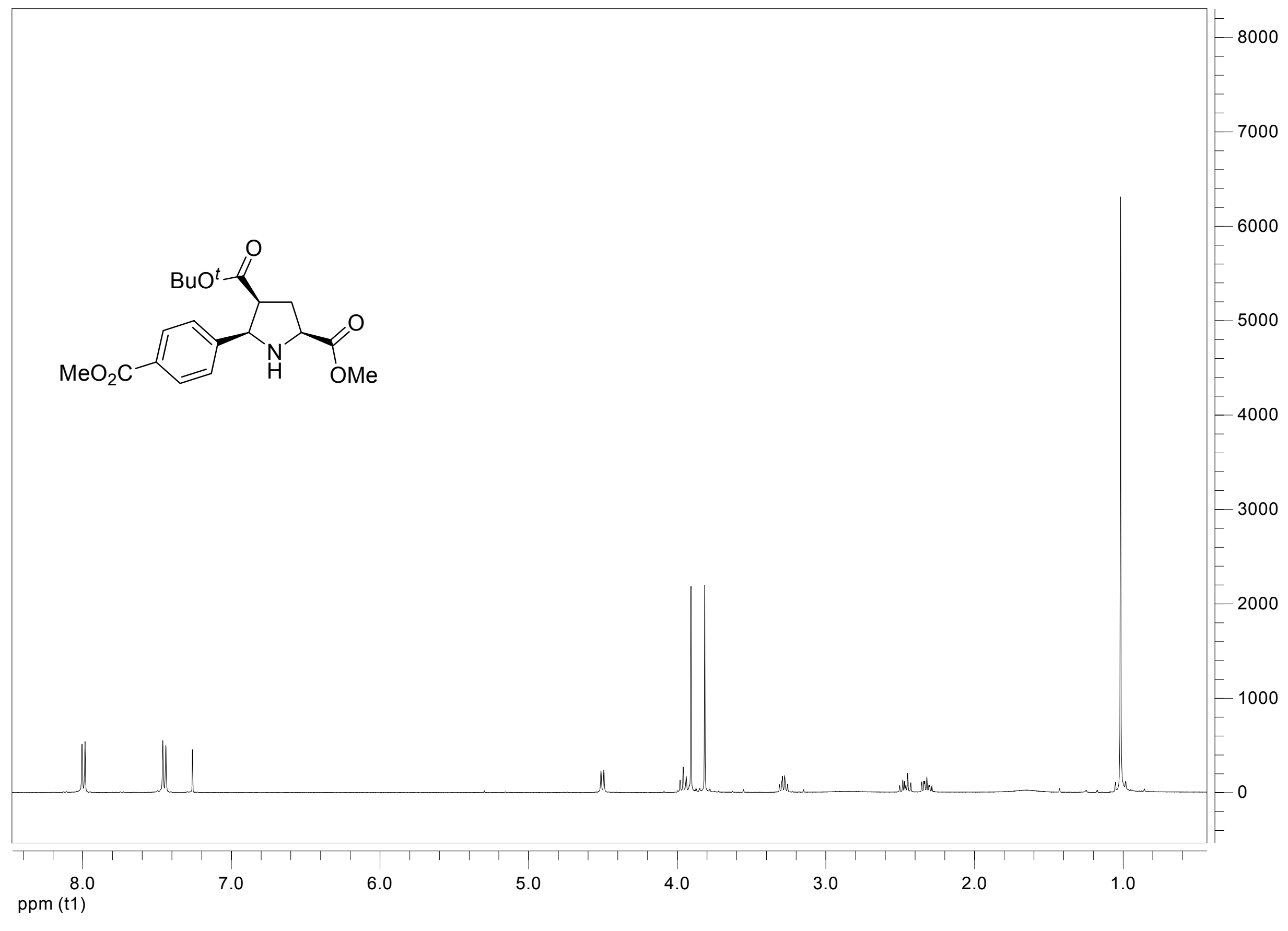




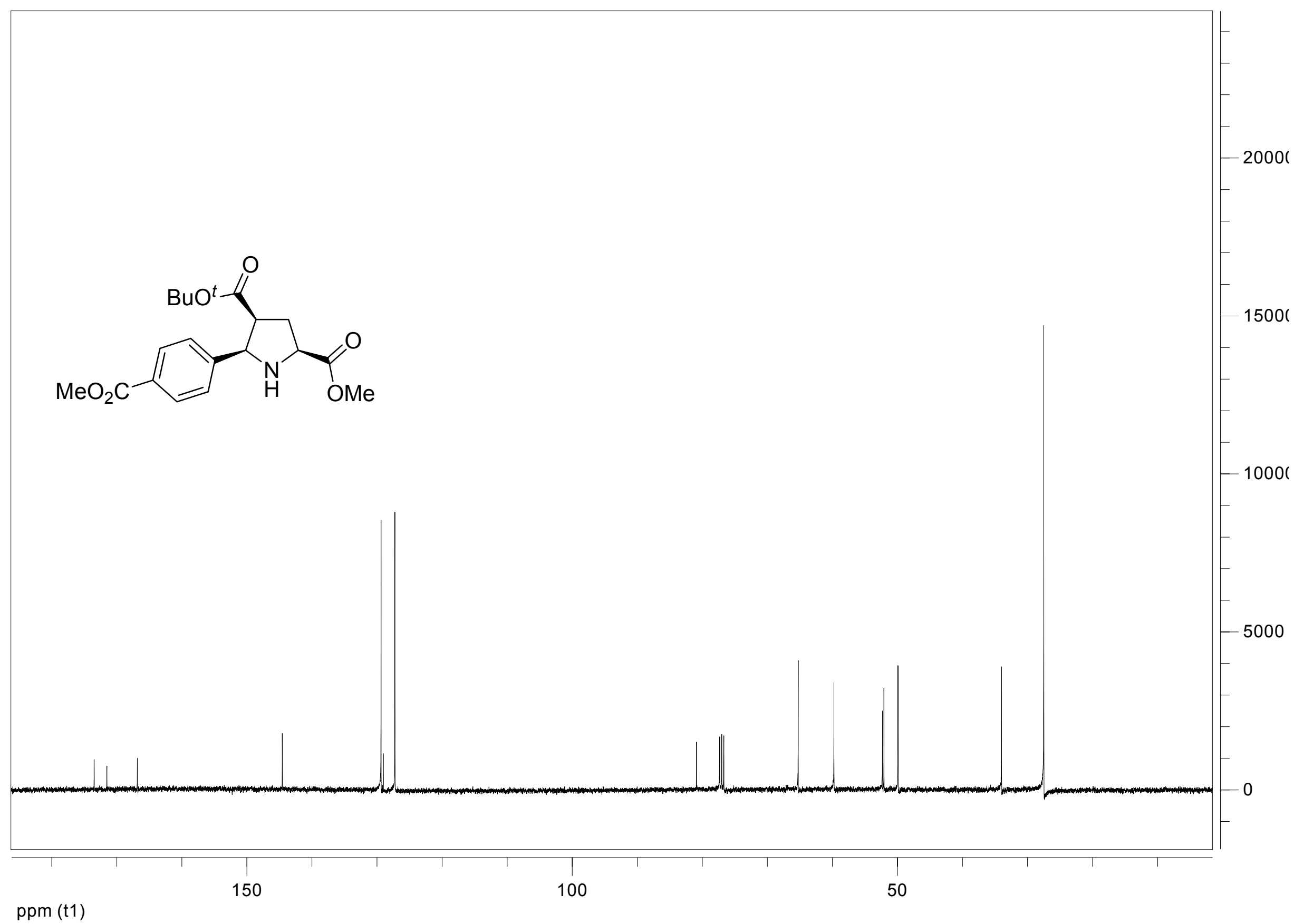




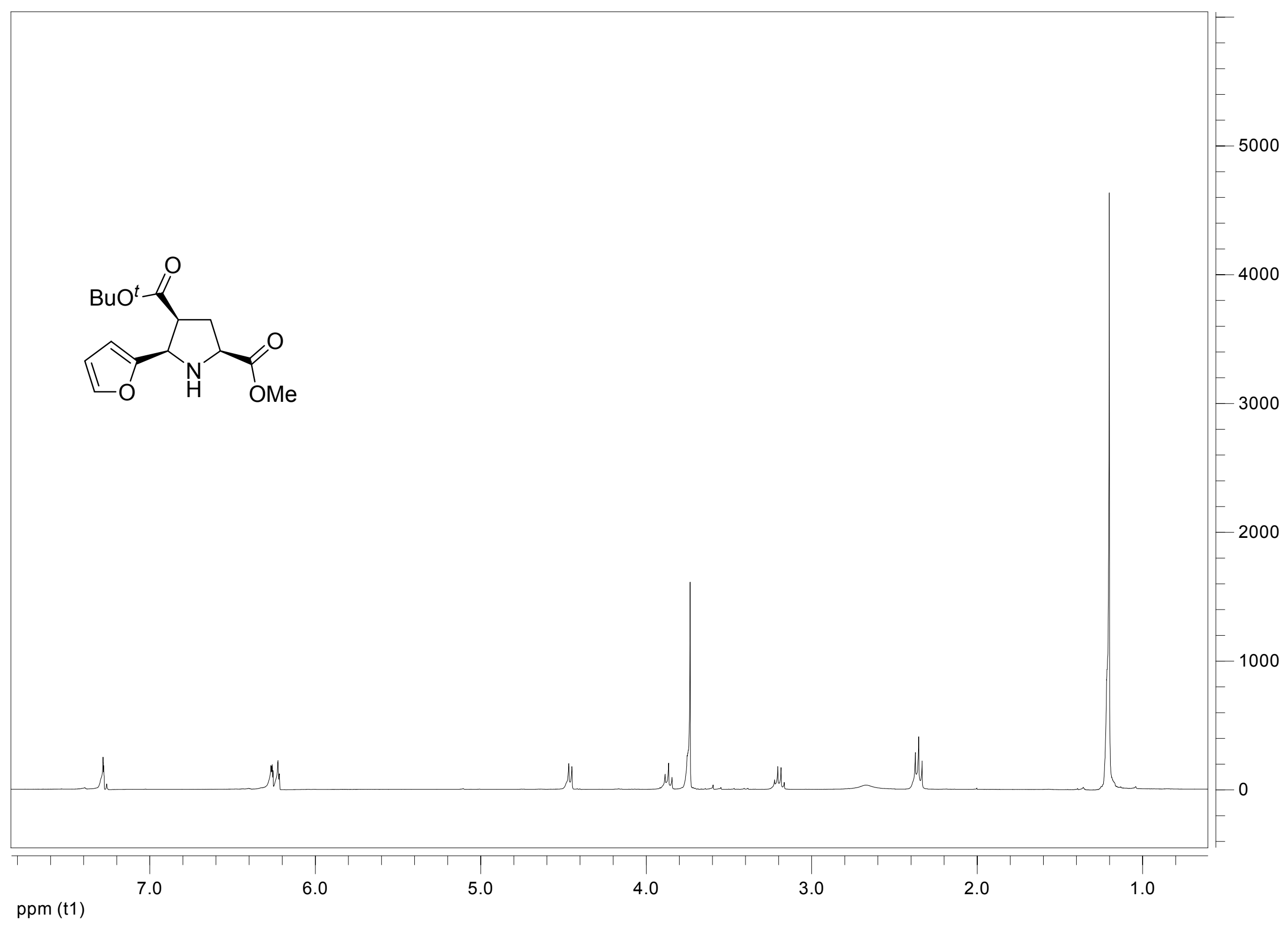




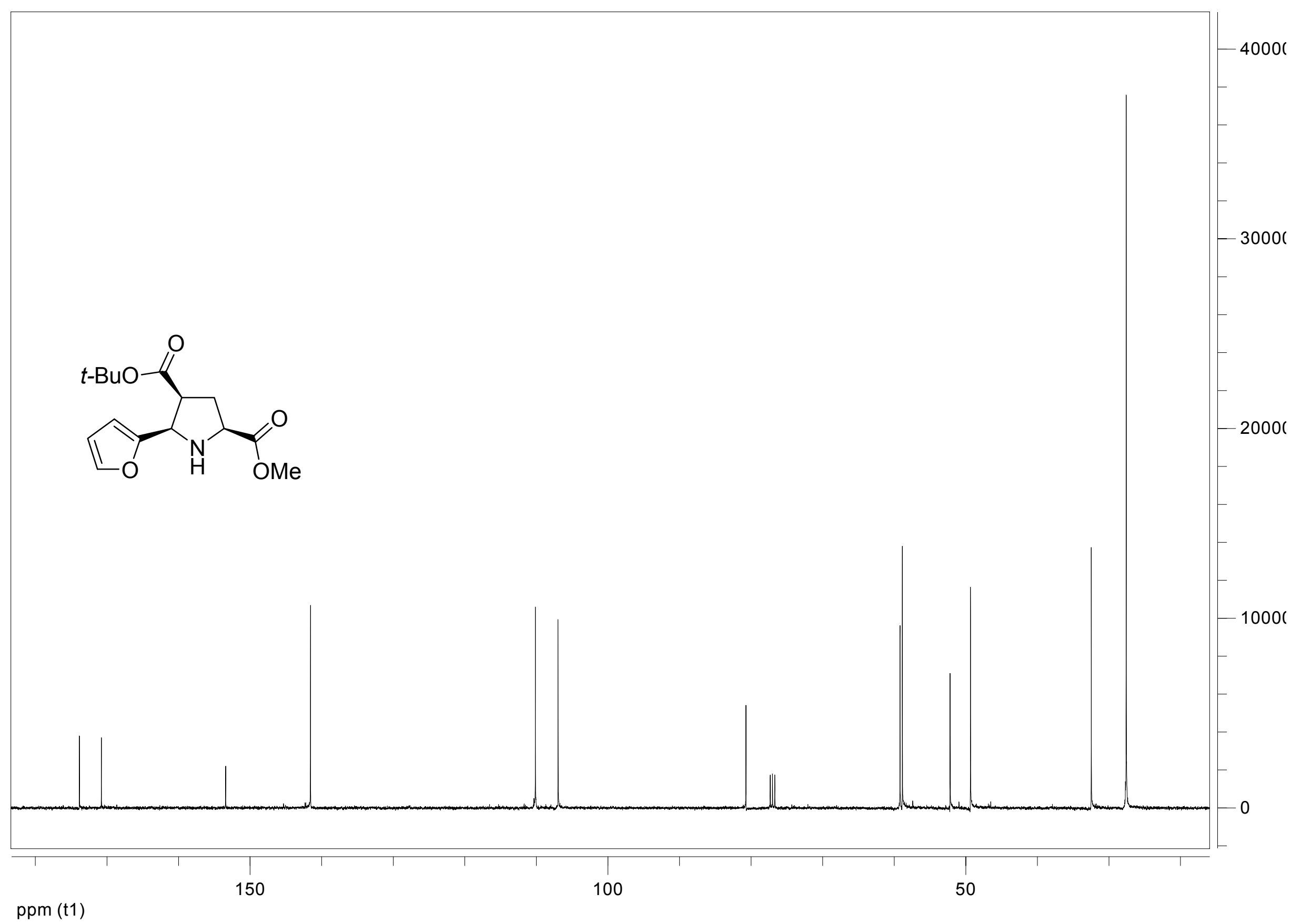




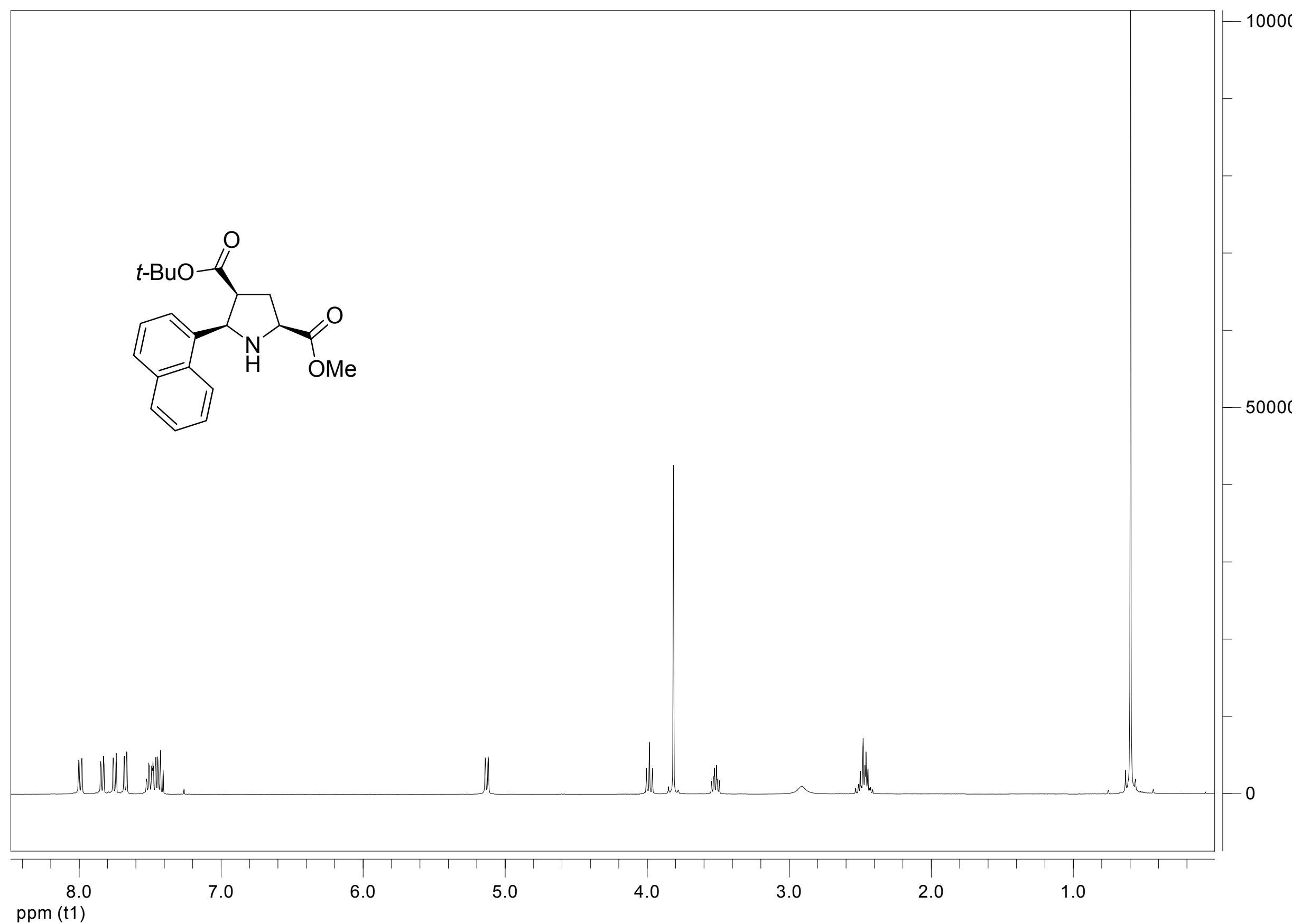




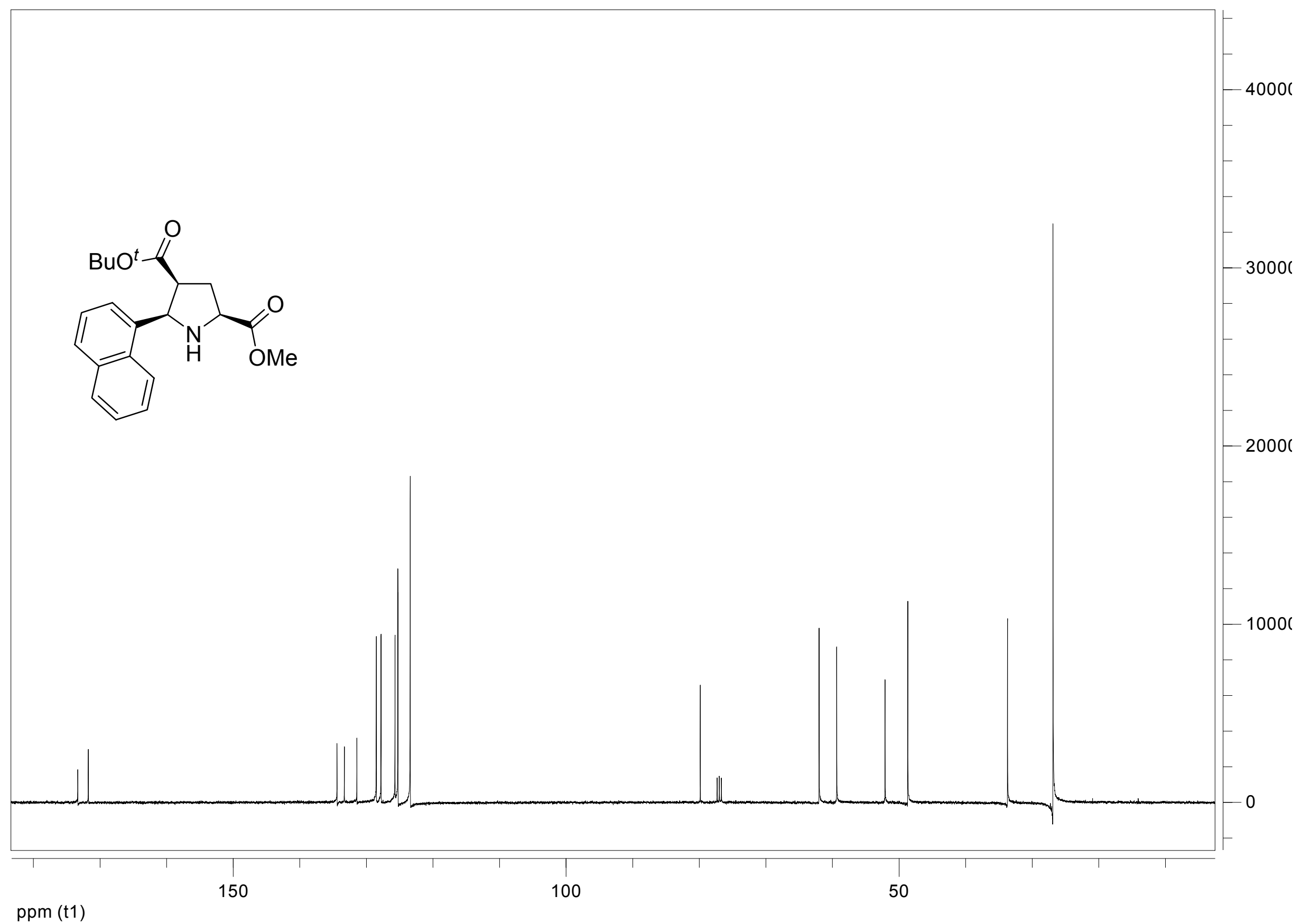




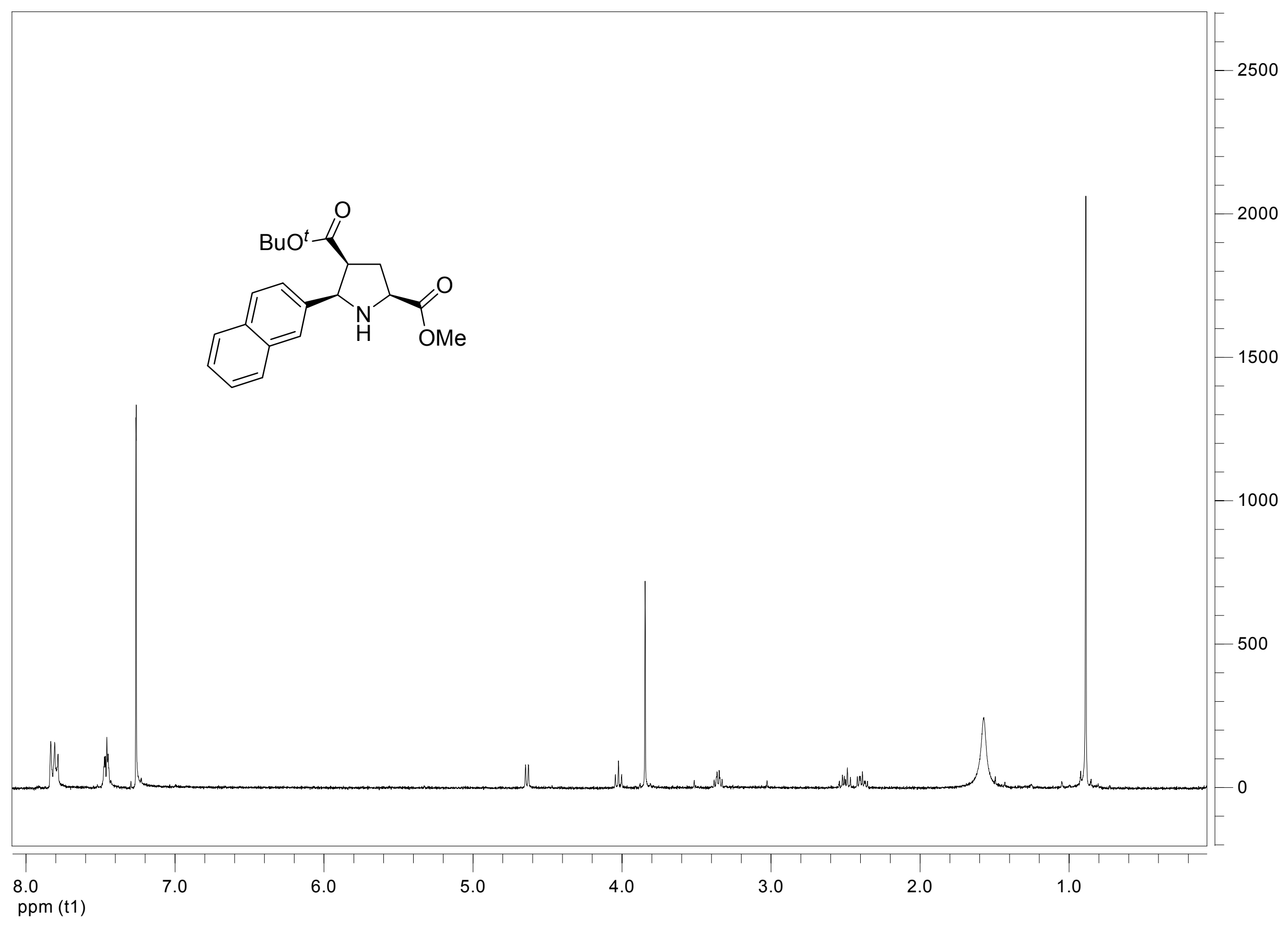




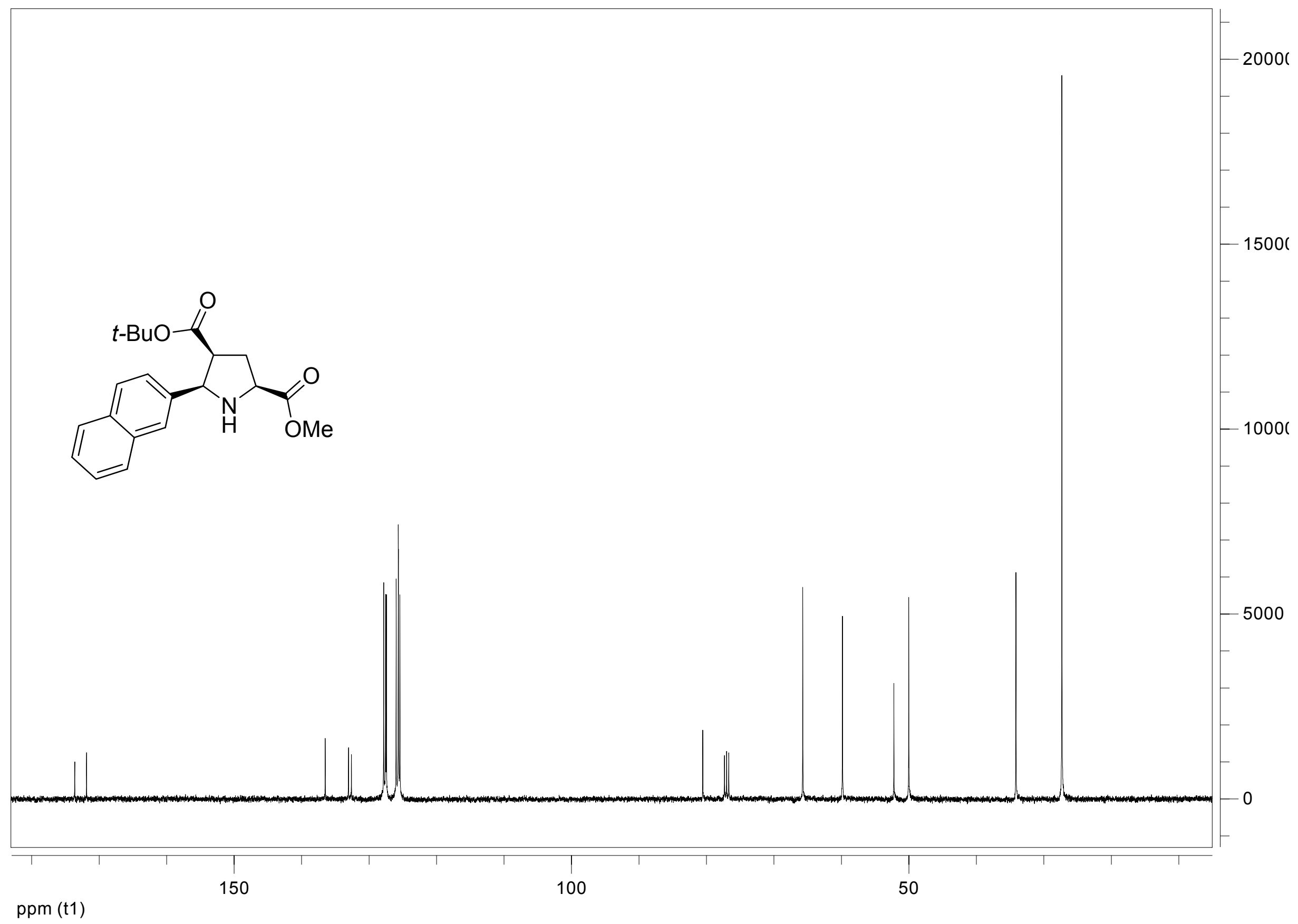




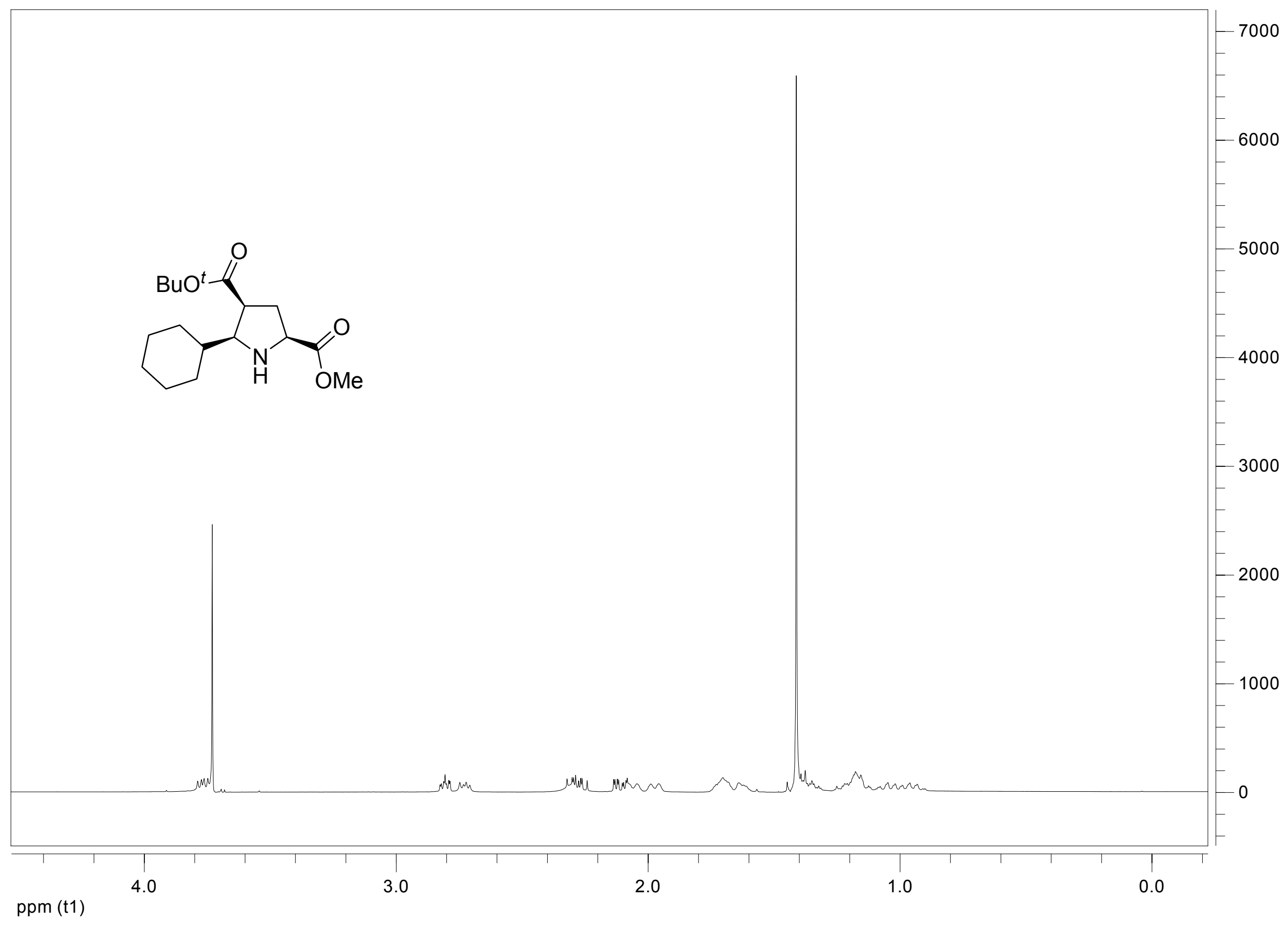




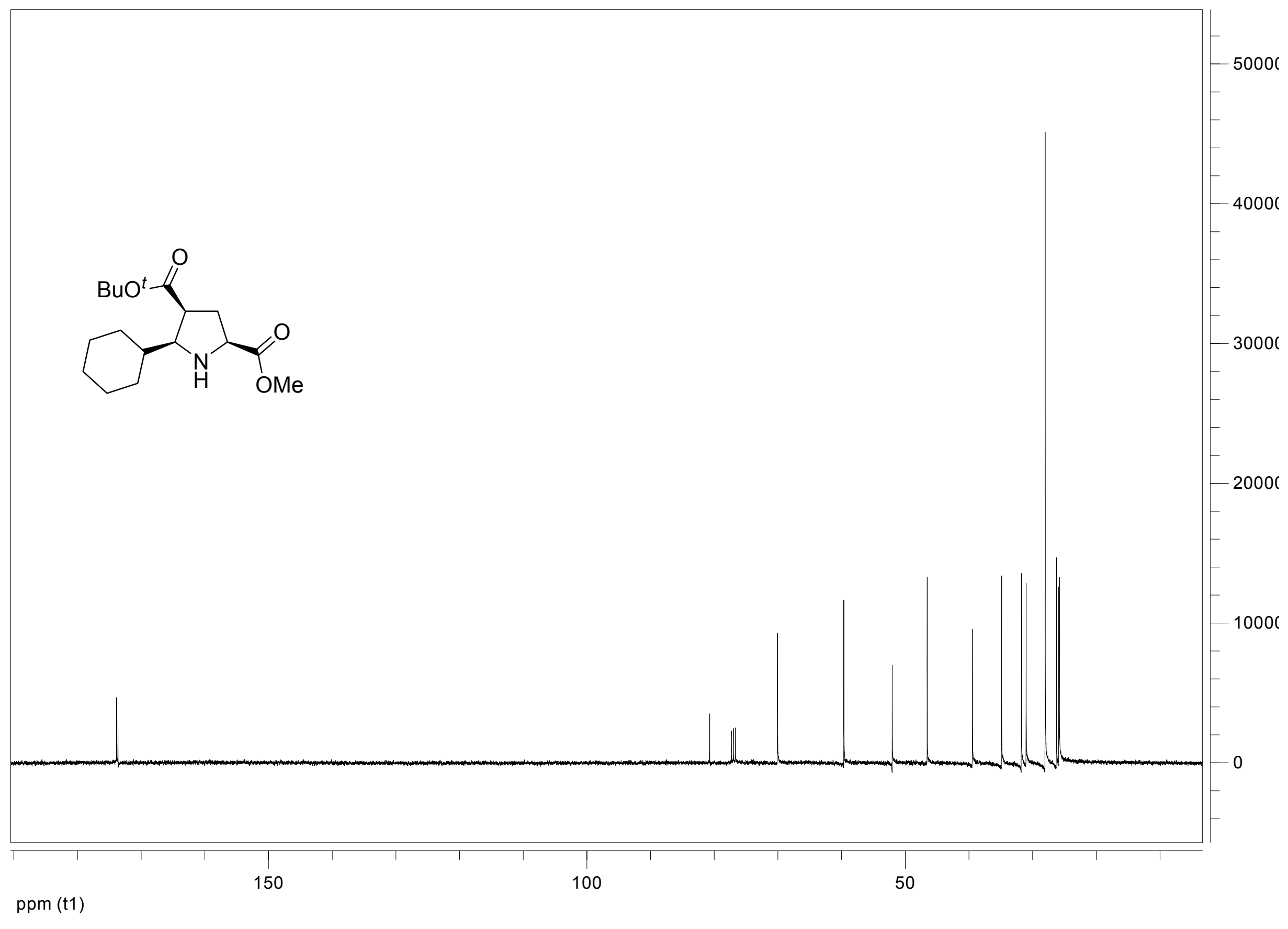




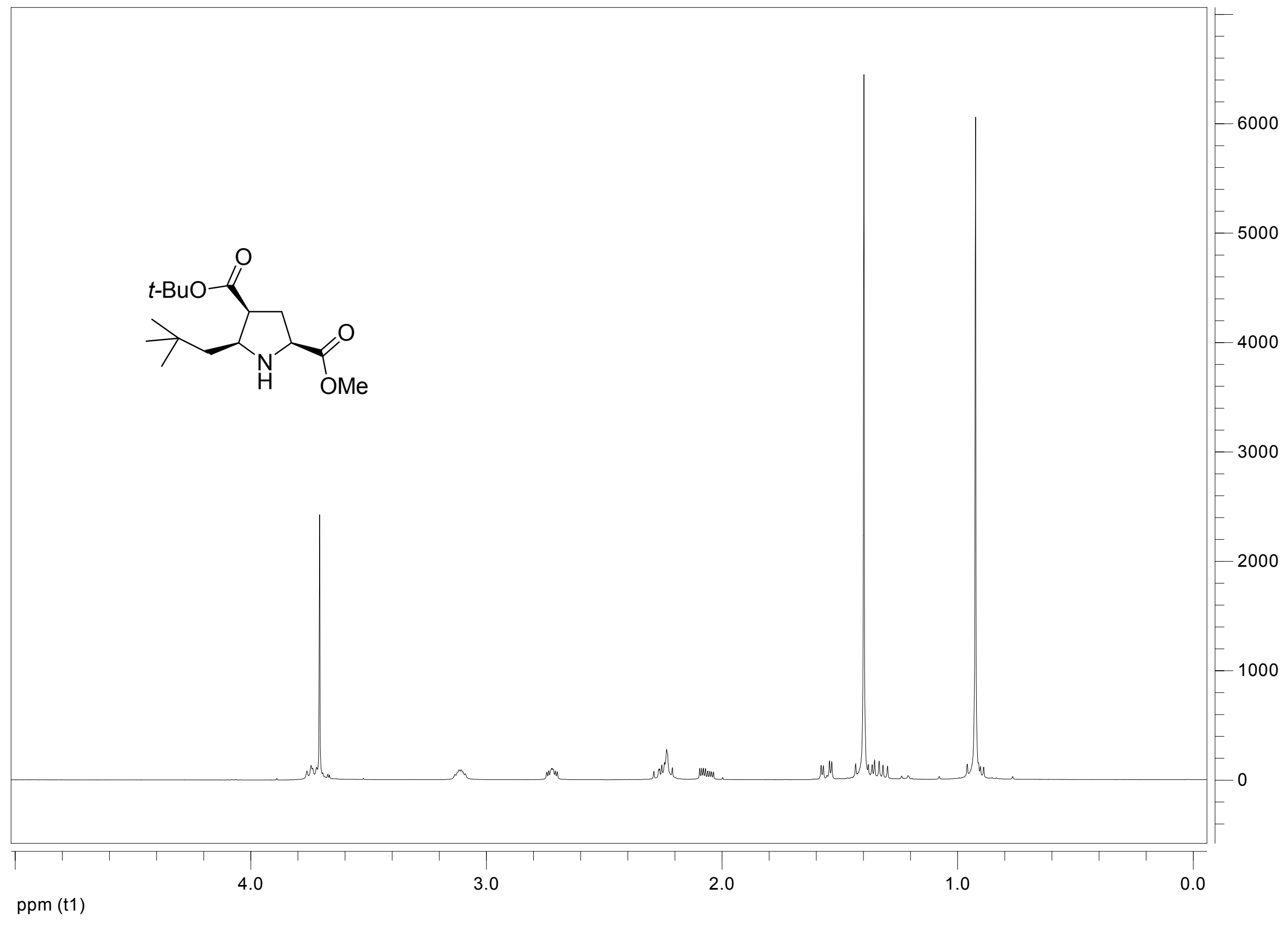




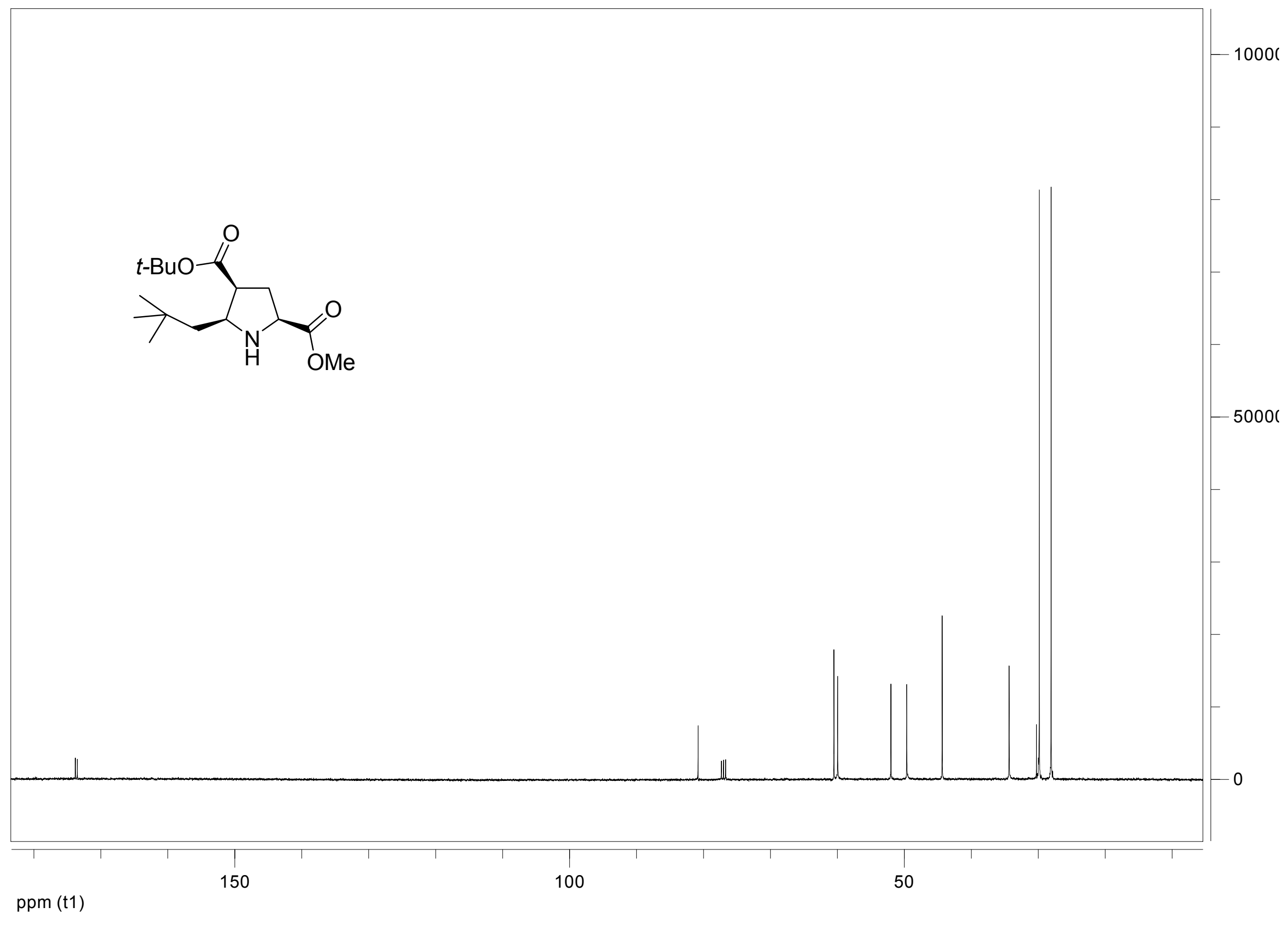




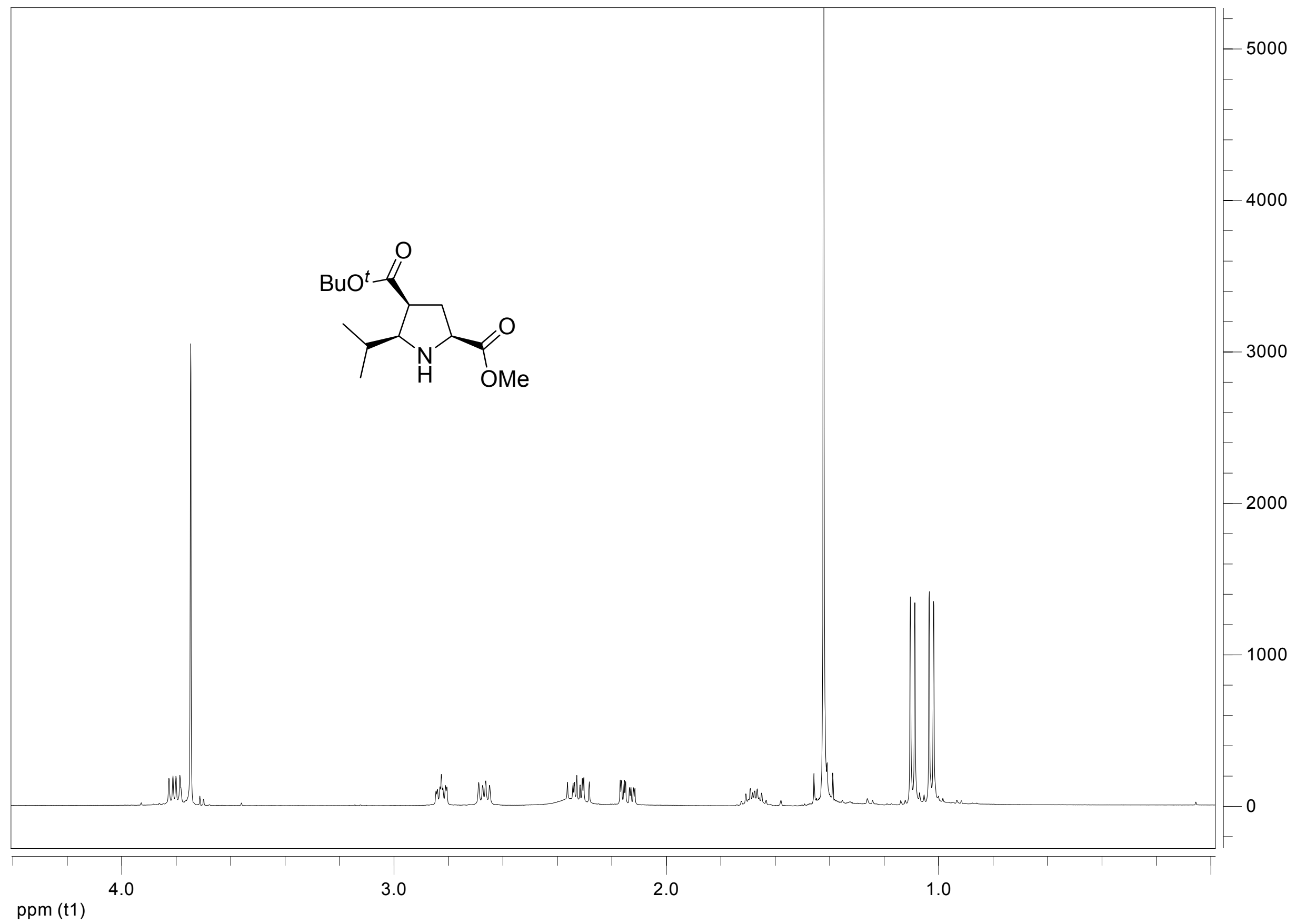




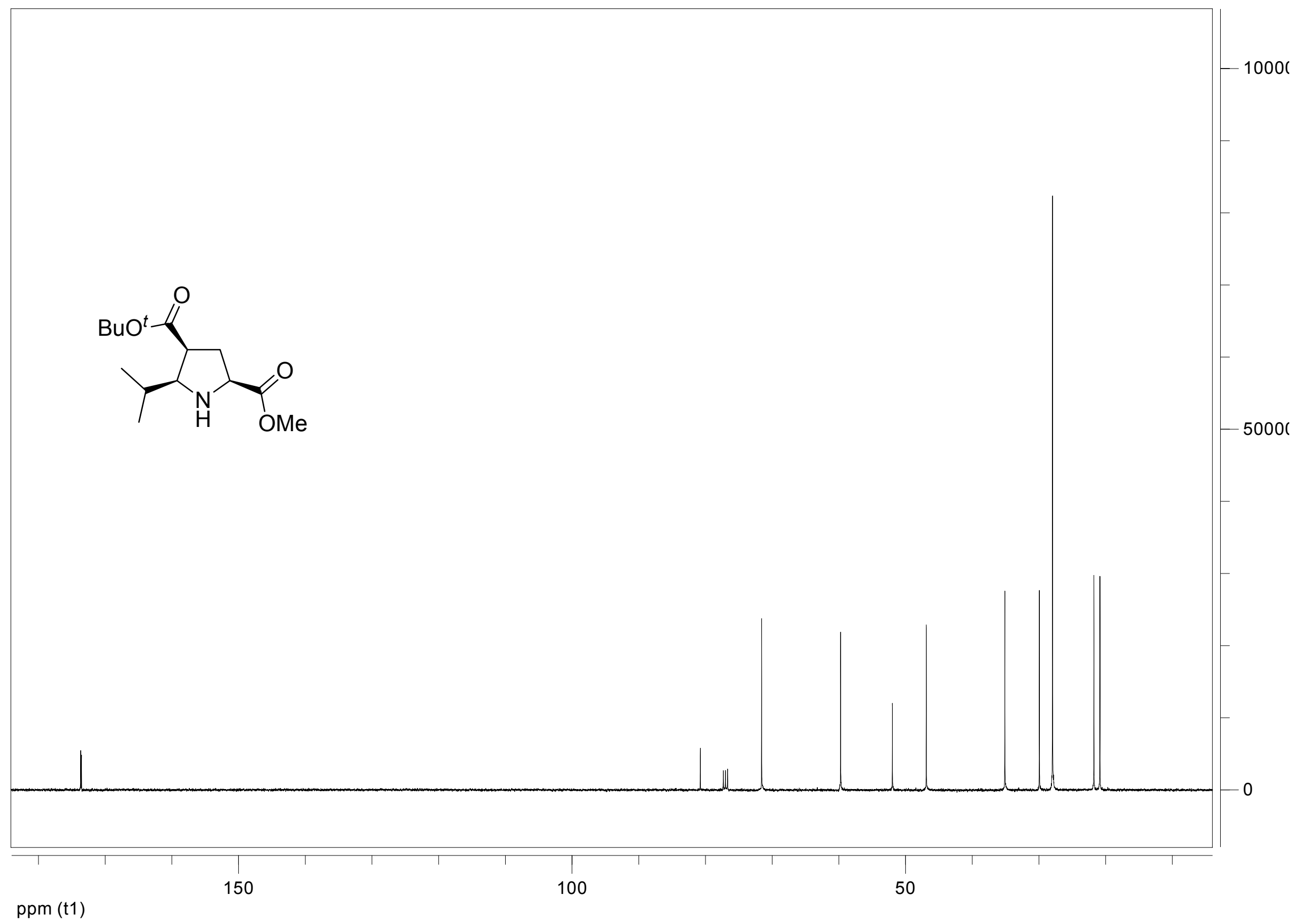

